$\mathrm{IC} / 2005 / 101$

SIAS-CMTP-05-2

USTC-ICTS-05-11

hep-th/0511004

\title{
Two Loop Effective Kähler Potential of (Non-)Renormalizable Supersymmetric Models
}

\author{
Stefan Groot Nibbelink ${ }^{a, 1}$, Tino S. Nyawelo ${ }^{b, 2}$, \\ a Shanghai Institute for Advanced Study, University of Science and Technology of China, \\ 99 Xiupu Rd, Pudong, Shanghai 201315, P.R. China \\ Interdisciplinary Center for Theoretical Study, University of Science and Technology of China, \\ Hefei, Anhui 230026, P.R. China \\ ${ }^{b}$ The Abdus Salam ICTP, Strada Costiera 11, I-34014 Trieste, Italy
}

\begin{abstract}
We perform a supergraph computation of the effective Kähler potential at one and two loops for general four dimensional $\mathcal{N}=1$ supersymmetric theories described by arbitrary Kähler potential, superpotential and gauge kinetic function. We only insist on gauge invariance of the Kähler potential and the superpotential as we heavily rely on its consequences in the quantum theory. However, we do not require gauge invariance for the gauge kinetic functions, so that our results can also be applied to anomalous theories that involve the Green-Schwarz mechanism. We illustrate our two loop results by considering a few simple models: the (non-)renormalizable Wess-Zumino model and Super Quantum Electrodynamics.
\end{abstract}

\footnotetext{
$1 \quad$ E-mail: nibbelin@ustc-sias.cn

2 E-mail: tnyawelo@ictp.it
} 


\section{Introduction and Summary}

The use of the effective action in quantum field theories has proven to be a very powerful tool. Following [1] in particular the quantum effective potential is widely used. (For a recent application of the computation of the vacuum energy in extra dimensions see [2].) The effective action of a supersymmetric theory up to two derivatives is encoded in three functions of the chiral multiplets: the Kähler potential, the superpotential and the gauge kinetic function. The latter two are required to be holomorphic and are therefore very much constraint. This is reflected in various non-renormalization theorems [3] for these functions and lead to results to all order $[4,5]$. The works $[6,7,8]$ show that even a lot of non-perturbative information can be obtained. In particular, in the certain $\mathcal{N}=2$ theories the full non-perturbative superpotential has been computed [9].

The Kähler potential on the other hand is only required to be a real function, and therefore far less constrained. It receives corrections at all orders in perturbation theory, unless the theory has sufficient amounts of extended supersymmetries. For example, the Kähler potential of the adjoint chiral multiplet in $\mathcal{N}=2$ Super Yang-Mills theory is determined by a prepotential that also fixes the gauge kinetic function. The computation of the Kähler potential at the one loop level has been performed by many authors $[10,11,12,13,14]$, and their results essentially agree with each other. One loop corrections the Kähler potential have also been computed in the context of supergravity $[15,16,17]$. An extension of such works to extra dimensions can be found in [18].

At the two loop level only some partial results have been obtained. The two loop Kähler potential for the renormalizable Wess-Zumino model with a cubic superpotential has been computed [19, 20, 21, 22], and has been extended to a general non-renormalizable Wess-Zumino model by some people from the same group [23, 24]. The latter result seem suspicious because it does not appear to be covariant. As far as we are aware no calculation of the two loop Kähler potential has been performed which takes the effect of gauge interactions into account.

Precisely because for the Kähler potential there are no non-renormalization theorems in general, it is interesting to understand it quantum corrections at higher orders. The computation of the effective Kähler potential can also be very important for phenomenological applications, as it encodes the wave function renormalization of chiral multiplets. The physical masses of chiral multiplets can be determined only when the effect of wave function renormalization is taken into account. Similarly if the gauge kinetic function is not a pure constant, then the wave function renormalization of the chiral multiplets that appear in the gauge kinetic function induce non-holomorphic renormalization of the gauge couplings. Also when one investigates the effective potential at the two loop level, knowing the effective Kähler potential might prove very useful. For some of these applications it might be relevant to know the renormalization of the superpotential at two loops. Here there is a somewhat surprising result that the superpotential is renormalized, but only by a finite amount [20, 21, 25]. At the two loop level, for the class of theories we are considering, the computation of the selfenergy of the chiral multiplets can easily involve over a hundred diagrams, which is very hard to manage. As we will see the computation of the effective Kähler potential only requires about eleven supergraphs to be evaluated. The complicated expressions for the selfenergies are recovered by evaluating the second mixed derivative of the two loop effective Kähler potential.

In this paper we perform supergraph computations of the Kähler potential up to two loops in generic globally supersymmetric theories. The explicit one and two loop formulae for the effective Kähler potential can be found in sections 3 and 4.4, respectively. The classical theory is not assumed to be renormalizable, and we allow for gauge interactions to be present. The only simplifying assumptions 
that we are making are that the classical theory has no more than second derivatives and the gauged symmetries are realized linearly ${ }^{3}$. Both our one and two loop results are manifestly target space diffeomorphism covariant. When we restrict to the ungauged case, we see that we obtain the same terms at two loops as $[23,24]$, but with different coefficients such that the result contains the curvature tensor and covariant derivatives of the superpotential. This shows that our results are manifestly covariant. The result of the two loop Kähler potential looks surprisingly simple as long as the gauge kinetic function is strictly constant.

Apart from the possible applications mentioned above, our results at the two loop level might be interesting for various applications in $\mathcal{N}=2$ theories. In theories with extend supersymmetry the Kähler and super-potential are obtained from a single holomorphic prepotential. Since our results are obtained for generic $\mathcal{N}=1$ supersymmetric theories, they can be applied in particular to $\mathcal{N}=2$ theories, and may provide important cross checks on the validity of the constraints that come from the $\mathcal{N}=2$ structure. At the one loop level such computations have been performed [11, 12]. Our results can be used to undertake a similar analysis at the two loop level.

Before we close the introduction and summary section, we would like to explain our methodology in the outline of the paper. In section 2 we present the basic starting point of our calculations: a general $\mathcal{N}=1$ supersymmetric model based on a Kähler manifold with some of its linear isometries gauged. As far as superspace is concerned we will be using the conventions of Wess and Bagger [26]. To compute the one and two loop corrections to the Kähler potential we employ a background field method. Since we are interested in the effective Kähler potential, we assume that the background consists of chiral multiplet. Since in this paper we are focusing on the quantum corrections to the Kähler potential only, we will be systematically ignoring all derivatives on the background chiral superfields in the whole project, as these terms constitute higher derivative operators. These higher derivative operators are beyond the scoop of the present paper. We do not assume that the background is constant, because then we are not able to extract the effective Kähler potential. In principle, we are computing the 1PI effective Kähler potential, however, to ensure that the relevant propagators do not become too complicated, we make use of the background equations of motion.

One consequence of this chiral background is that the gauge symmetry is generically spontaneously broken. The resulting quadratic mixing between the quantum vector and chiral multiplets makes quantization awkward. To overcome this complication we remove this mixing by using a supersymmetric generalization of the 't Hooft $R_{\xi}$ gauge fixing procedure. A consequence of this gauge is that the ghost action becomes dependent on the background chiral multiplets and cannot be neglected. Moreover, in this gauge the Goldstone bosons and the ghosts have equal mass eigenvalues. We will be making a special choice for the $R_{\xi}$ gauge fixing parameter to have the maximal simplification of the super propagators. Because of this we loose the explicit dependence on a gauge fixing parameter and therefore we are not able to trace any possible gauge dependence of our results.

In section 3 we review the computation of the one loop Kähler potential by summing bubble to explain our procedure to reduce supergraph to scalar graphs. We use standard supergraph techniques that can be found in the textbooks $[27,28]$. To regularize the theory we employ the dimensional reduction scheme $(\overline{\mathrm{DR}})[29,30]$. (Following arguments summarized in refs. [31, 32] we assume that for our purposes the $\overline{\mathrm{DR}}$ scheme is fully consistent.) A well-known consequence of this scheme is that quadratic divergences do not show up explicitly. (This will also be the case at the two loop level,

\footnotetext{
${ }^{3}$ Because it is easy to recognize the linear Killing vectors in all our expressions, it would be straightforward to generalize them to arbitrary non-linear gaugings.
} 
therefore our results cannot be directly compared to those of [33].) Our one loop results are consistent with results obtained by previous groups $[10,11,12,13,14]$, as long as we do not consider non-Abelian gauge interactions. The difference in that case is presumably a consequence of the fact that those results are obtained in the Landau gauge while we use a supersymmetric variant of the 't Hooft $R_{\xi}$ gauge.

The core of our paper is contained in section 4 where we perform our two loop computation. We first compute a single diagram of the "8" topology which quickly reduces to a product of two one-loop scalar graphs. After that we turn to the other nine two-loop supergraphs with the " $\ominus$ " topology. Some of them have the property that they partly turn into "8" scalar graphs. The resulting scalar graphs are evaluated in appendix B. We summarize the results of the contributions to the Kähler potential at two loop in subsection 4.4, and compare with the partial results that exist in the literature on two loop effective Kähler potential calculations.

Since our results are written in terms of some tensor valued integrals, it is useful to see how the formulae can be applied to some simple examples. In section 5 we discuss both the non-renormalizable Wess-Zumino model and its renormalizable limit. Super Quantum Electrodynamics constitutes our second example.

\section{Acknowledgments}

We would like to thank T. Hirayama and R. Zwicky for pleasant discussions at various stages of this project. We grateful to A. Brignole for communications on the one loop Kähler potential. We have benefitted from correspondence with C. Ford, I. Jack and D.R.T. Jones concerning the computation of two loop scalar graphs. We are also grateful to S.J. Tyler for pointing out a missing contribution and some typos in the published version of this work.

\section{Setup: $\mathcal{N}=1$ gauge non-linear sigma model}

We consider a general $\mathcal{N}=1$ supersymmetric non-linear sigma model of (anti-)chiral superfields $\phi^{a}\left(\bar{\phi}_{\underline{a}}\right)$ described by a Kähler potential $K(\bar{\phi}, \phi)$ and a superpotential $W(\phi)$. The indices $a$ and $\underline{a}$ label the chiral and anti-chiral multiplets, respectively. Some of the linear isometries $\delta_{\alpha} \phi=i \alpha \phi=i \alpha^{I} T_{I} \phi$ (where $\alpha_{I} \in \mathbb{R}$ are gauge parameters) are assumed to be gauged by the introduction of the non-Abelian gauge vector superfield $V=V^{I} T_{I}$. The Hermitean generators $T_{I}$ of this group satisfy the algebra $\left[T_{I}, T_{J}\right]=c^{K}{ }_{I J} T_{K}$, with purely imaginary structure coefficients $c^{K}{ }_{I J}$. The Killing metric is denoted by $\delta_{I J}$. We write tr and Tr for the traces over the representation of the chiral multiplets $\phi$ and the adjoint representation, respectively. Gauging is of course only possible if the Kähler potential and the superpotential are gauge invariant

$$
K\left(\bar{\phi} e^{-i \alpha}, e^{i \alpha} \phi\right)-K(\bar{\phi}, \phi)=0, \quad W\left(e^{i \alpha} \phi\right)=W(\phi) .
$$

This will have important consequences for the quantum interactions. Unlike the Kähler potential or the superpotential, the gauge kinetic function $f_{I J}(\phi)$ need not be gauge invariant, as it may play a role in a Green-Schwarz mechanism to cancel anomalies.

The central task of this paper is to compute quantum corrections to the Kähler potential at one and two loops. To obtain the functional dependence of one and two loop corrections on chiral multiplets, we expand the theory around some non-trivial background $\phi$ for the chiral multiplets, while assuming 
a trivial background for the gauge sector. We do not need a non-trivial gauge background because the appearance of the vector multiplet in the effective Kähler potential can be reconstructed by gauge invariance. The background $\phi$ is assumed to fulfill the classical equations of motion. To expand around this background we make the replacement $\phi \rightarrow \phi+\Phi$, and declare that the vector superfield $V$ and the chiral superfield $\Phi$ are quantum fields. Only the quantum fields appear in the loops, while the background fields encoded the external legs.

For generic non-renormalizable models also higher derivative operators will be required as counter terms. Since we only focus on the computation of the quantum effective Kähler potential not the full effective quantum action, we will be ignoring all (super covariant) derivatives $\left(D_{\alpha}, \bar{D}_{\dot{\alpha}}\right.$ and $\left.\partial_{m}\right)$ on the background fields. It is irrelevant whether these derivatives arises from partial integrations or the use of the background equations of motion. Let us stress, that this does not mean that we treat $\phi$ as a strictly constant background. Because if we would take $\phi$ constant, any superspace integral over them will vanish, including the effective Kähler potential which we seek to compute. But it does mean that in our computation we are allowed to ignore the super derivative terms of the background equations of motion

$$
-\frac{1}{4} \bar{D}^{2} K_{a}+W_{a}=0, \quad-\frac{1}{4} D^{2} K^{\underline{a}}+\bar{W}^{\underline{a}}=0
$$

because they would give rise to higher derivative operators. (Here differentiations w.r.t. the background variables $\phi$ and $\bar{\phi}$ are denoted as $K_{a}=K_{, a}, K^{a}=K^{a}$, etc.) Therefore, for our purposes we may use that $W_{a}=0$, even though the field value of $\phi$ does not need to satisfy this supersymmetric vacuum condition.

In the expansion around the background $\phi$ and in the computation of the one and two loop effective Kähler potential we run into various geometrical quantities, which we introduce here. The Kähler metric is defined as $G \underline{a}_{a}=K \underline{a}_{a}=K \underline{a}_{a}$; with $G^{-1}$ we indicate its inverse. Given the metric we can construct other geometrical quantities like the connections

$$
\Gamma_{a b}^{c}=K_{a b} \underline{\underline{c}} G_{\underline{c}}^{-1 c}, \quad \bar{\Gamma}^{\underline{a}} \underline{\underline{b}}=K^{\underline{a}} \underline{\underline{b}}{ }_{c} G_{\underline{c}}^{-1 c},
$$

and the curvature

$$
R^{\underline{a}} \underline{b}_{b}=K^{\underline{a} \underline{b}}{ }_{a b}-K^{\underline{a}} \underline{b}_{c} G^{-1 c}{ }_{\underline{c}} K_{a b} \underline{\underline{c}} .
$$

Using the connections we can define covariant derivatives $W_{; a b \ldots}$ of the superpotential $W$. As explained around (2) we may set $W_{a}=0$ to find

$$
W_{; a b}=W_{a b}, \quad W_{; a b c}=W_{a b c}-\Gamma_{a b}^{d} W_{d c}-\Gamma_{b c}^{d} W_{d a}-\Gamma_{c a}^{d} W_{d b} .
$$

This completes the classical geometrical description of the globally supersymmetric linearly-gauged non-linear sigma model.

To define the perturbative quantum theory we perform the expansion in quantum field $\Phi$ and $V$ around the background $\phi$. The non gauge fixed action for the quantum fields $\Phi$ and $V$ is composed of the (classical) Kähler term

$$
S_{K}=\frac{1}{2} \int \mathrm{d}^{8} z\left\{K\left(\bar{\phi}+\bar{\Phi}, e^{2 V}(\phi+\Phi)\right)+K\left((\bar{\phi}+\bar{\Phi}) e^{2 V}, \phi+\Phi\right)+\xi \operatorname{tr} V\right\}
$$


with the superspace measure $\mathrm{d}^{8} z=\mathrm{d}^{4} x \mathrm{~d}^{4} \theta$, a Fayet-Iliopoulos parameters $\xi$, the superpotential interactions

$$
S_{W}=\int \mathrm{d}^{6} z W(\phi+\Phi)+\int \mathrm{d}^{6} \bar{z} \bar{W}(\bar{\phi}+\bar{\Phi})
$$

and finally the gauge kinetic part

$$
S_{G}=\frac{1}{4} \int \mathrm{d}^{6} z \operatorname{tr} f_{I J}(\phi+\Phi) \mathcal{W}^{I \alpha} \mathcal{W}_{\alpha}^{J}+\frac{1}{4} \int \mathrm{d}^{6} z \operatorname{tr} \bar{f}_{I J}(\bar{\phi}+\bar{\Phi}) \overline{\mathcal{W}}_{\dot{\alpha}}^{I} \overline{\mathcal{W}}^{J} \dot{\alpha}
$$

with the chiral measure $\mathrm{d}^{6} z=\mathrm{d}^{4} x \mathrm{~d}^{2} \theta$ and is conjugate. The gauge kinetic function $f_{I J}$ is symmetric in the gauge indices $I$ and $J$. Its real part $h_{I J}=\frac{1}{2}\left(f_{I J}+\bar{f}_{I J}\right)$ is equal to the gauge coupling $1 / g^{2}$. A non-constant gauge kinetic function appears for example in the effective models from string theory, in particular when they rely on the Green-Schwarz mechanism to cancel anomalies. The super gauge field strengths are given by

$$
\mathcal{W}_{\alpha}=-\frac{1}{8} \bar{D}^{2}\left(e^{-2 V} D_{\alpha} e^{2 V}\right), \quad \overline{\mathcal{W}}_{\dot{\alpha}}=-\frac{1}{8} D^{2}\left(\bar{D}_{\dot{\alpha}} e^{2 V} e^{-2 V}\right)
$$

with the notational convention that operators, like $D_{\alpha}$, only act on the first superfield on the right. We have given a representation of the gauged Kähler potential action (6) which is manifestly real.

From these expression we determine the propagators of the various superfields. Because of the super gauge invariance the kinetic operator of the vector multiplet is not invertible. This requires gauge fixing and the introduction of the corresponding supersymmetric Faddeev-Poppov ghosts $C, C^{\prime}, \bar{C}, \bar{C}^{\prime}$, see e.g. $[27,28]$. For the general supersymmetric theories under consideration in this paper, two additional complications arise: Firstly, the background $\phi$ spontaneously breaks some of the gauge symmetries, and therefore leads to quadratic mixing of the vector multiplets $V$ with the chiral multiplets $\Phi$ and $\bar{\Phi}$. To avoid this mixing the gauge fixing functional $\Theta^{I}$ can be modified to

$$
\Theta^{I}=-\frac{\sqrt{2}}{4} \bar{D}^{2}\left(V^{I}+\left(h^{-1}\right)^{I J} K^{\underline{a}}{ }_{a}\left(T_{J} \phi\right)^{a} \frac{1}{\square} \bar{\Phi}_{\underline{a}}\right) .
$$

This is very similar to the 't Hooft $R_{\xi}$ gauge fixing for spontaneously broken gauge theories. The gauge fixing procedure is then implemented in the standard way by the insertion of

$$
\Delta_{F P}\left|\delta\left(\Theta^{I}-F^{I}\right)\right|^{2} e^{i S_{F}}, \quad S_{F}=\int d^{8} z h_{I J} \bar{F}^{I} F^{J},
$$

in the path integral defining the quantum theory, where $\Delta_{F P}$ is the Faddeev-Poppov determinant and $F^{I}$ are arbitrary chiral multiplets. The second complication is that the Gaussian integral over the gauge fixing chiral variables $F^{I}$ and $\bar{F}^{I}$ involves the function $h_{I J}$ which is in general a function of $\phi$ and its conjugate. Therefore, we need to properly normalize this Gaussian integral. This can be implemented by the introduction of the Nielsen-Kallosh ghosts $\chi^{I}$, which have the same quadratic action as $F^{I}$, but they are anti-commuting.

The gauge fixing procedure together with the introduction of the various ghosts leads to the addition of the following terms to the classical action. First of all, the gauge fixing term is obtained by performing the functional integral over $F^{I}$ :

$$
S_{G F}=-\int \mathrm{d}^{8} z h_{I J} \bar{\Theta}^{I} \Theta^{J}
$$


with $\Theta^{I}$ given in (10). This seems to be worrying because the presence of $1 / \square$ leads to non-local interaction. However, as far as the definition of the perturbation theory is concerned this non-locality leads to perfectly well defined propagators. The Faddeev-Poppov ghost action reads

$$
S_{F P}=\frac{1}{\sqrt{2}} \int \mathrm{d}^{6} z C_{I}^{\prime} \delta_{C} \Theta^{I}+\frac{1}{\sqrt{2}} \int \mathrm{d}^{6} \bar{z} \bar{C}_{I}^{\prime} \delta_{C} \bar{\Theta}^{I} .
$$

Here $\delta_{C} \Theta^{I}$ denotes the gauge transformation of the gauge fixing functional to first order in the super gauge parameters

$$
\delta_{\Lambda} \Theta^{I}=\sqrt{2} \frac{\bar{D}^{2}}{-4}\left\{\bar{\Lambda}^{I}+[V, \Lambda-\bar{\Lambda}]^{I}-2\left(h^{-1}\right)^{I J}\left(T_{J} \phi\right)^{a} K^{\underline{a}_{a}} \frac{1}{\square}(\bar{\phi} \bar{\Lambda}+\bar{\Phi} \bar{\Lambda})_{\underline{a}}\right\}+\ldots,
$$

but with the super gauge parameter $\Lambda^{I}$ replaced by the ghosts $C^{I}$, etc. The dots denote higher order terms in $V$ which are not relevant for our computations. To obtain this we have made use of the super gauge transformations

$$
\bar{\Phi} \rightarrow(\bar{\phi}+\bar{\Phi}) e^{-2 \bar{\Lambda}}-\bar{\phi}, \quad e^{2 V} \rightarrow e^{2 \bar{\Lambda}} e^{2 V} e^{2 \Lambda}, \quad \Phi \rightarrow e^{-2 \Lambda}(\phi+\Phi)-\phi
$$

linearized in the (anti-)holomorphic super gauge parameters $(\bar{\Lambda}) \Lambda$. Because the background generically spontaneously breaks the gauge symmetry, the gauge transformations of the quantum fields $\Phi$ are non-linear. Finally, the Nielsen-Kallosh ghost action

$$
S_{N K}=-\int \mathrm{d}^{8} z h_{I J} \bar{\chi}^{I} \chi^{J}
$$

completes the full action of the quantum theory.

We now determine the propagators by reading off quadratic parts of the quantum action. As the Nielsen-Kallosh ghost action (16) is quadratic by itself we do not need to discuss it again. The quadratic actions of the vector and Faddeev-Poppov ghost superfields are given by

$$
\begin{aligned}
& S_{V}^{2}=-\int \mathrm{d}^{8} z V^{I}\left[h \square-M_{V}^{2}\right]_{I J} V^{J}, \\
& S_{F P}^{2}=\int \mathrm{d}^{8} z\left(C_{I}^{\prime}\left[\mathbb{1}-h^{-1} M_{C}^{2} \frac{1}{\square}\right]_{J}^{I} \bar{C}^{J}+\bar{C}_{I}^{\prime}\left[\mathbb{1}-h^{-1} M_{C}^{2 T} \frac{1}{\square}\right]_{J}^{I} C^{J}\right) .
\end{aligned}
$$

Here we have introduced the Hermitean mass matrices

$$
\left(M_{C}^{2}\right)_{I J}=2 \bar{\phi} T_{I} G T_{J} \phi, \quad M_{V}^{2}=\frac{1}{2}\left(M_{C}^{2}+M_{C}^{2}\right)
$$

for the ghost and vector multiplets. Because $M_{C}^{2 T}$ refers to the transpose of $M_{C}^{2}$ (i.e. its vector indices $I, J$ are interchanged), the matrix $M_{V}^{2}$ is symmetric. By construction of the gauge fixing (10) and (12) there is no mixing between the vector and the chiral multiplet, instead the quadratic part of the chiral multiplet action has become more complicated

$$
S_{\Phi}^{2}=\int \mathrm{d}^{8} z\left(\bar{\Phi}_{\underline{a}}\left[G-G M_{G}^{2} \frac{1}{\square}\right]_{a}^{\underline{a}} \Phi^{a}+\frac{1}{2} \Phi^{a} W_{a b} \frac{D^{2}}{-4 \square} \Phi^{b}+\frac{1}{2} \bar{\Phi}_{\underline{a}} \bar{W}^{\underline{a}} \underline{\underline{b}} \frac{\bar{D}^{2}}{-4 \square} \bar{\Phi}_{\underline{b}}\right)
$$




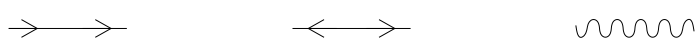

Figure 1: Here we depict the super propagators that will be employed to computed the quantum corrections to the Kähler potential. The first two diagrams correspond to the chiral propagators defined in (22): The first one represents $\Delta_{\Phi \Phi}$ and the second one $\Delta_{\Phi \Phi}$. The latter two refer to the vector and Faddeev-Poppov ghost propagators given in (21), respectively.

where we introduce (partly for later reference) the superpotential, Goldstone and total mass matrices

$$
M_{W}^{2}=G^{-1} \bar{W}\left(G^{-1}\right)^{T} W, \quad\left(M_{G}^{2}\right)^{a}{ }_{b}=2\left(T_{I} \phi\right)^{a}\left(h^{-1}\right)^{I J}\left(\bar{\phi} T_{J} G\right)_{b}, \quad M^{2}=M_{W}^{2}+M_{G}^{2},
$$

respectively. We use the matrix notation $W$ to denote the second derivative of the superpotential: $[W]_{a b}=W_{, a b}$ for notational convenience.

From the quadratic expressions for the gauge and ghost multiplets it is straightforward to determine the resulting superfield propagators. Even though the quadratic action of the ghost multiplets (17) have non-local terms, the resulting propagators are well-behaved

$$
\Delta_{V V}=\left[h \square-M_{V}^{2}\right]^{-1}, \quad \Delta_{C^{\prime} \bar{C}}=\left[\square-h^{-1} M_{C}^{2}\right]^{-1}, \quad \Delta_{\bar{C}^{\prime} C}=\left[\square-h^{-1} M_{C}^{2 T}\right]^{-1} .
$$

The non-locality of (19) is also not reflected in the chiral multiplet propagators

$$
\begin{gathered}
\Delta_{\bar{\Phi}}=\left[\square-M_{G}^{2}\right]^{-1} G^{-1}+\left[\square-M_{W}^{2}\right]^{-1} G^{-1}-G^{-1} \frac{1}{\square}=\left[\square-M^{2}\right]^{-1} G^{-1}, \\
\Delta_{\Phi \Phi}=G^{-1}\left[\square-M^{2}\right]^{-1} \bar{W}\left(G^{-1}\right)^{T}, \quad \Delta_{\bar{\Phi} \Phi}=\left(G^{-1}\right)^{T} W G^{-1}\left[\square-M^{2}\right]^{-1} .
\end{gathered}
$$

In supergraphs the propagators $\Delta_{\Phi \Phi}$ and $\Delta_{\bar{\Phi}}$ are multiplied by $-D^{2} /(4 \square)$ and $-\bar{D}^{2} /(4 \square)$, respectively. (In figure 1 we have collected our graphical representation for these propagators.) Because of super gauge invariance of the superpotential (1), the superpotential matrix $W$ has zero modes $T_{I} \phi$ : $W_{a b}\left(T_{I} \phi\right)^{b}=0$, up to derivative terms which we ignore in the computation of the effective Kähler potential using the background equations of motion. By combining this observation with the definition of the masses in (20) we arrive at the propagators (22). These zero modes correspond to the Goldstone modes of the global symmetries generated by $T_{I}$. This implies that $M_{W}^{2} M_{G}^{2}=M_{G}^{2} M_{W}^{2}=0$, and consequently that three propagators in the first equation of (22) can be combined into one, and that $M^{2}$ can be replaced by $M_{W}^{2}$ in the last two equations of (22).

This can be interpreted as follows: The background defined by $\phi$ generically leads to spontaneous symmetry breaking and massive vector multiplets. The corresponding massless Goldstone superfields are eaten by these massive vector superfields in the unitary gauge. However, we have chosen a different gauge, defined by (10) and (12), to ensure the absence of mixing between the vector and the chiral multiplets. In this gauge a massive vector multiplet consists of $V^{I}$ (with $I$ such that $T_{I} \phi \neq 0$ ), the Goldstone mode chiral superfields (identified by $T_{I} \phi$ ), and the massive Faddeev-Poppov ghosts $C_{I}, C_{I}^{\prime}$. Moreover, in this gauge the chiral Goldstone multiplets and the ghost multiplets have the same mass eigenvalues, because for any integral power $p$ we have

$$
\operatorname{tr}\left(M_{G}^{2}\right)^{p}=\operatorname{Tr}\left(h^{-1} M_{C}^{2}\right)^{p}=\operatorname{Tr}\left(h^{-1} M_{C}^{2 T}\right)^{p} .
$$

Moreover, the symmetric part of the ghost mass is equal to the vector multiplet mass, see (18). This leads to partial cancellations of the contributions of the Goldstone chiral multiplets and the FaddeevPoppov ghosts in the one loop computation of the Kähler potential, as we will see in the next section. 


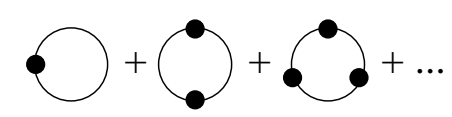

Figure 2: At the one loop level the effective Kähler potential is obtained from summing an infinite set of one loop bubble diagrams. This corresponds to the computation of various functional determinants.

\section{One loop effective Kähler potential}

The one loop calculation of the effective Kähler potential involves the computation of one loop vacuum bubble graphs with multiple insertions of two-point interaction terms, as indicated in figure 2. The definition of the two point interaction $\mathcal{M}$ and the propagator $\Delta$ here is somewhat arbitrary. To see why this is actually convenient, consider for example, a chiral multiplet with both a mass term and a quadratic Kähler term, which are both functions of the background fields. To evaluate the corresponding Gaussian integral, we can first take the mass as a two point interaction and compute in the way explained below. The remaining Gaussian integral with a Kähler metric between the quantum chiral superfields is evaluated by taking the identity matrix to define the propagator, and the Kähler metric minus the identity as the two point interaction. Hence, it is precisely this freedom in the choice of the propagator and the two point interaction that helps to make convenient choices to evaluate the Gaussian path integrals effectively.

To evaluate these bubbles in general, we consider a generic vector of commuting superfields $\Psi$ with quadratic action

$$
S=\frac{1}{2} \int \mathrm{d}^{8} z \Psi^{T}\left[\Delta^{-1}+\mathcal{M}\right] \Psi
$$

The sum of the connected bubble graphs reads

$$
i \Gamma=\frac{1}{2} \sum_{n \geq 1} \frac{(-)^{n}}{n} \int\left(\mathrm{d}^{8} z\right) \ldots \operatorname{tr}\left(\mathcal{M}_{1} X_{\underline{1} 1} \Delta_{\underline{1} 2} \mathcal{M}_{2} X_{\underline{2} 2} \Delta_{\underline{2} 3} \ldots \mathcal{M}_{n} X_{\underline{n} n} \Delta_{\underline{n} 1}\right),
$$

where $1,2, \ldots, n$ and $\underline{1}, \underline{2}, \ldots, \underline{n}$ are labels that denote the various superspace coordinates $z_{1}, z_{2}, \ldots, z_{n}$ and $z_{1}, z_{2}, \ldots, z_{\underline{n}}$. The corresponding measure is represented by $\left(\mathrm{d}^{8} z\right) \ldots$. By functional differentiation w.r.t. sources $J$ that couple to $\Psi$, we have obtained

$$
X_{\underline{11}}=\frac{\delta J_{\underline{1}}}{\delta J_{1}}=\frac{\delta J\left(z_{1}\right)}{\delta J\left(z_{1}\right)}=\delta_{\underline{11}} .
$$

Here $\delta_{\underline{11}}=\delta_{\underline{11}}^{4} \delta^{4}\left(\theta_{1}-\theta_{1}\right)$ denotes the superspace delta function, and $\delta_{\underline{11}}^{4}=\delta^{4}\left(x_{1}-x_{1}\right)$ the four dimensional spacetime delta function. (When the sources correspond to chiral multiplets, we find some additional super covariant derivatives hitting the superspace delta function.) When we consider anti-commuting superfields, the Faddeev-Popov and Nielsen-Kallosh ghosts, the expression in (25) has one additional overall minus sign.

We can apply this to the various quadratic terms derived in the previous section. Using various superspace and supergraph techniques we finally find that the full one loop Kähler potential is given by

$$
i \Gamma_{1 L}=\int\left(\mathrm{d}^{4} x\right)_{12} \mathrm{~d}^{4} \theta\left[\operatorname{Tr} \ln h+\operatorname{Tr} \ln \left(\mathbb{1}-\frac{h^{-1} M_{C}^{2}}{\square}\right)-\operatorname{tr} \ln G-\frac{1}{2} \operatorname{tr} \ln \left(\mathbb{1}-\frac{M_{W}^{2}}{\square}\right)\right]_{1} \delta_{12}^{4} \frac{1}{\square_{1}} \delta_{12}^{4},
$$




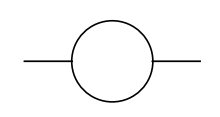

Figure 3: This one loop self energy supergraph is computed as a cross check for the one loop Kähler potential determined in this section.

in a coordinate representation. In accordance with a general non-renormalization theorem of supersymmetry [3, 28], this expression is local in the Grassmann variables. This is because of the superspace expression becomes proportional to $A_{2} \delta_{12}\left[B D^{2} \bar{D}^{2}\right]_{2} \delta_{12}=16 A_{2} \delta_{12}^{4} B_{2} \delta_{12}^{4} \delta^{4}\left(\theta_{1}-\theta_{2}\right)$, where $A$ and $B$ are some operators which may be functions of spacetime derivatives $\partial_{m}$. The origins of the various terms in (27) are as follow: The first term is due to the Nielsen-Kallosh ghosts. The second term is the combined effective action of the Faddeev-Poppov ghosts and the Goldstone chiral multiplets, using that their mass eigenvalues in the gauge (10) are all equal, see (23). (The bubble graphs with vector multiplets vanish identically in this gauge, because no $D, \bar{D}$ ever hit any superspace delta functions.) The last two terms are due bubbles that contain chiral multiplets.

As it stands this expression (27) is ill-defined and requires regularization. Mainly because computational convenience at the two loop level, we have chosen to use dimensional reduction [29, 30]. In appendix A we have collected the one loop integrals calculated in this scheme. A peculiar consequence of the $\overline{\mathrm{DR}}$ scheme is, that both the first and the third term are absent, as follows from (A.1). Using (A.3) and (A.5), and dropping the $1 / \epsilon$ poles, we find that the remaining terms result in the effective one loop Kähler potential

$$
K_{1 L}=-\frac{1}{16 \pi^{2}} \operatorname{Tr} h^{-1} M_{C}^{2}\left(2-\ln \frac{h^{-1} M_{C}^{2}}{\bar{\mu}^{2}}\right)+\frac{1}{32 \pi^{2}} \operatorname{tr} M_{W}^{2}\left(2-\ln \frac{M_{W}^{2}}{\bar{\mu}^{2}}\right)
$$

where $\bar{\mu}^{2}=4 \pi e^{-\gamma} \mu^{2}$ defines the $\overline{\mathrm{MS}}$ renormalization scale, and the mass matrices $M_{C}^{2}$ and $M_{W}^{2}$ are defined in (18) and (20), respectively. To use the expressions from appendix A for matrices, we can first diagonalize the mass matrices, and apply these expressions for the eigenvalues, and after that transform back to the original basis.

To concluded the discussion concerning the one loop effective Kähler potential, we would like to mention an important consistency check of our computation and compare our results to the existing literature. The effective Kähler potential can be used to determine the wave function renormalization at one loop by taking the second mixed derivative of it. This wave function renormalization can also be computed directly from the supergraph given in figure 3. For this cross check it is sufficient to consider the renormalizable Wess-Zumino model (see section 5.1 for details). The self energy supergraph gives the wave function renormalization

$$
\Sigma_{\text {self energy }}=-\frac{|\lambda|^{2}}{32 \pi^{2}} \ln \frac{|m+\lambda \phi|^{2}}{\bar{\mu}^{2}}
$$

which agrees with our one loop effective Kähler potential result:

$$
\Sigma_{\text {eff. Kähler pot. }}=\frac{\partial^{2} K_{1 L}}{\partial \phi \partial \bar{\phi}}=-\frac{|\lambda|^{2}}{32 \pi^{2}} \ln \frac{|m+\lambda \phi|^{2}}{\bar{\mu}^{2}},
$$

see (54). As long as no non-Abelian gauge interaction are taken into account, our one loop results are consistent with some existing literature concerning the computation of the effective Kähler potential 


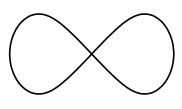

a

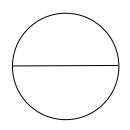

$\mathrm{b}$

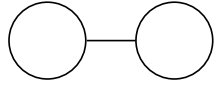

$\mathrm{c}$

Figure 4: There are three basic vacuum graphs at the two loop level. They have the topologies of an "8" (figure a) and " $\ominus$ " (figure b), a "double tadpole" (figure c), respectively.

to that order $[10,11,12,25,13,14]$. In the non-Abelian case the mass matrices $M_{C}^{2}$ and $M_{V}^{2}$ are not equal anymore, and our results slightly deviate from these reference. This might be an artifact of the use of different gauge fixing procedures.

\section{Two loop effective Kähler potential}

At the two loop level there are three different topologies of the supergraphs that may contribute to the Kähler potential. They are depicted in figure 4. The third topology is one-particle-reducible and can be ignored when computing the effective Kähler potential as we will argue in subsection 4.1. In the following two subsections we first reduce the supergraphs to scalar momentum integrals. Then using the evaluation of these momentum integrals in appendix B, we can give the renormalized expressions for these scalar integrals in the $\overline{\mathrm{DR}}$ scheme. We collect our final results in subsection 4.4.

We obtain the vertices, from which the "8" and " $\ominus$ " graphs can be derived, by expanding the various actions given in section 2 up to fourth order in the quantum fields. The four point vertices are relevant for the "8" supergraphs, while " $\ominus$ " supergraphs can be build from two three-point interactions. We list only the relevant vertices for diagrams that do not vanish automatically. When performing these expansions in the quantum fields, it is important to take the consequences of gauge invariance (1) of the Kähler potential $K$ and the superpotential $W$ into account. Because none of the propagators (21) and (22) mix with each other, we can exclude many diagrams, which would be present in many other gauges. In addition we have the constraint, that supergraphs are only nonvanishing at two loop if they involve an equal number of super covariant derivatives $D$ and $\bar{D}$, and of each of them at least four. Chiral and ghost multiplets will introduce a combination of $D^{2}$ and $\bar{D}^{2}$ into the diagram, while the vector multiplets do not. Therefore, the number of $D^{2}$ and $\bar{D}^{2}$ of graphs with vector superfields will in general be lower than the ones involving only chiral multiplets, and hence such graphs are more likely to vanish. As we will see in the subsequent subsections such considerations reduce the number of relevant diagrams, to a manageable number for manual computations.

\section{1 "Double tadpole" supergraphs}

Most computations of the effective (Kähler) potential are restricted to only those connected graphs that are one-particle-irreducible. The argument for this restriction is that all one-particle-reducible connected graphs contain one or more tadpole subgraphs, which are generically absent by symmetry arguments. (For example, a $\phi^{4}$ theory has the symmetry $\phi \rightarrow-\phi$ which forbids tadpoles to arise.) Because we are dealing with rather generic supersymmetric models in arbitrary backgrounds, we reconsider the issue of one-particle-reducible graphs.

For the computation of the effective Kähler potential at two loops there is only a single oneparticle-reducible topology, which has been depicted in figure 4.c. The connecting line can represent 


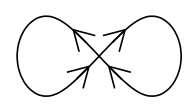

Figure 5: This is the only not necessarily vanishing supergraph of the " 8 " topology.

either a chiral or a vector multiplet propagators, while the loop lines may also refer to Faddeev-Poppov ghost multiplets. We can divide these diagrams into two classes depending on whether the connecting line is a chiral or a vector multiplet. In the case that the connecting line is a chiral superfield, one can show by some partial integrations of $D^{2}$ or $\bar{D}^{2}$ within the diagram and using relations like $D^{2} \bar{D}^{2} D^{2}=16 \square D^{2}$, that these diagrams contain too little $D^{2}$ or $\bar{D}^{2}$, and therefore vanish.

This leaves us with double tadpole graphs (figure 4.c) with a vector multiplet as a connecting line. Because a vector multiplet is not chiral, no $D^{2}$ or $\bar{D}^{2}$ appear on the connecting line. This implies that these double tadpole graphs are non-vanishing iff the sum of Fayet-Illiopoulos tadpole graphs a vector superfield is non-zero. The conditions for this have been well-studied in the literature (see e.g. ref. [34] and Weinberg's third volume [35]). Let us briefly review the arguments which are applicable in our case: If the vector multiplet is non-Abelian no tadpole is possible because the tadpole graph is never gauge invariant because of higher order (vector multiplet $V$ dependent) Lie derivatives acting on the chiral and anti-chiral gauge parameters $\Lambda$ and $\bar{\Lambda}$. For a $\mathrm{U}(1)$ vector superfield $V$ a tadpole (see (6)) is possible provided that the Fayet-Iliopoulos parameter $\xi$ is not a function of any (background) superfields. (Otherwise, again, the tadpole is not gauge invariant.) In particular, the induced $\xi$ at the one loop level is a constant, which is proportional to the sum of charges of all massless charged chiral superfields times the integral $\int \mathrm{d}^{4} p / p^{2}$. Since in this work we use dimensional reduction throughout, this integral vanishes. Therefore we conclude that the double tadpole graphs do not give any contribution to the effective Kähler potential at two loops in the $\overline{\mathrm{DR}}$ scheme.

\subsection{Supergraphs of the " 8 " topology}

The arguments presented in the first part of this section imply that there is in fact only one "8" supergraph (see figure 5) that results from the vertex

$$
\Delta S^{4} \supset \int \mathrm{d}^{8} z \frac{1}{4} K_{a b} \underline{a} \underline{b} \Phi^{a} \Phi^{b} \bar{\Phi}_{\underline{a}} \bar{\Phi}_{\underline{b}}
$$

which is obtained from the Kähler potential (6). Using standard supergraphs techniques we find that the supergraph, figure 5 , becomes the following scalar integral

$$
\left.i \Gamma_{2 L}^{\text {"8⿱口 }}=-\frac{i}{2} \int\left(\mathrm{d}^{4} x\right)_{123} \mathrm{~d}^{4} \theta K_{1 a b} \underline{a} \underline{b} \delta_{21}^{4}\left(\Delta_{\Phi \bar{\Phi}}\right)_{2}^{a} \underline{a}_{21} \delta_{31}^{4} \delta_{3 \bar{\Phi}}^{4}\right)_{3 \underline{b}}^{b} \delta_{31}^{4},
$$

with the chiral superfield propagator given in (22). The subscript ' 1 ' on the fourth mixed derivative of the Kähler potential emphasizes that it is evaluated in coordinate system '1'. For the other subscripts we refer back to section 3 .

By doing a Fourier transform to momentum space we see that also the corresponding scalar integral has the "8" topology. Because we need to do a double Wick rotation to Euclidean space, the expression changes sign. The resulting scalar integral $J$ defined in (B.1) is evaluated in appendix B. However, here the masses in the propagators are matrices rather than ordinary numbers. This does not pose a problem because we can simply reinterpret the formula for matrix valued masses. (This can be 


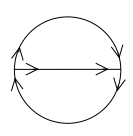

A

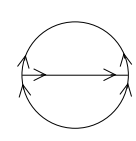

B

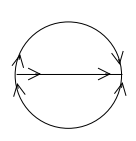

C

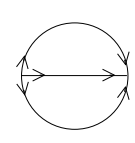

D

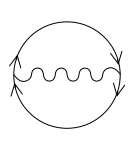

E

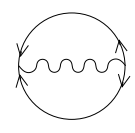

F

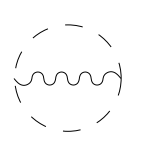

G

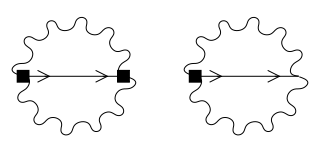

H

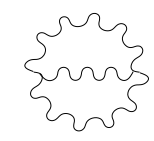

J

Figure 6: These are all the non-vanishing supergraphs of the " $\ominus$ " topology that can be obtained from the interaction (34). The arrows indicate whether chiral or anti-chiral legs are attached a vertex. If there are only chiral lines at a vertex and all their arrows are all either pointing inwards or outward, the vertex is a superpotential interaction. A thick square vertex refers to interactions that arise from the gauge kinetic functions. All other interactions involve the Kähler potential.

confirmed by performing a diagonalization of the masses after which the results of the appendix B can be readily used.) Concretely, the "8" supergraph can be compactly expressed as

$$
i \Gamma_{2 L}^{\text {"8" }}=\frac{i}{2} \int \mathrm{d}^{8} z K^{\underline{a}} \underline{b} a \bar{J}^{a} \underline{\underline{a}} \underline{b}\left(M^{2}, M^{2}\right),
$$

where the mass matrix $M^{2}$ is given by (20) and $\bar{J}^{a}{ }_{\underline{a}}{ }^{b} \underline{b}$ is defined in (B.22). Notice that, this expression is not covariant. This signals that this result is not complete. In the next section we see that covariance is restored by contributions coming from " $\ominus$ " supergraphs.

\subsection{Supergraphs of the " $\ominus$ " topology}

Next we consider the more complicated diagrams of the " $\ominus$ " topology. We first list the three point vertices that can give rise to non-vanishing supergraphs of this topology. From the Kähler potential (6), superpotential (7), the gauge kinetic terms (8) and the Faddeev-Poppov ghosts (13) and (14) we obtain

$$
\begin{aligned}
& \Delta S_{K}^{3} \supset \int \mathrm{d}^{8} z\left\{K_{\underline{a}_{a}}(V \Phi)^{a}(\bar{\Phi}+2 \bar{\phi} V)_{\underline{a}}+K_{a b^{\underline{a}}} \Phi^{b}\left((\bar{\phi} V)_{\underline{a}}(\Phi+2 V \phi)^{a}+\bar{\Phi}_{\underline{a}}\left(V \phi+\frac{1}{2} \Phi\right)^{a}\right)\right\}+\text { h.c. } \\
& \Delta S_{G}^{3} \supset-\int \mathrm{d}^{8} z\left\{\frac{1}{8} f_{I J} \bar{D}^{2} D^{\alpha} V^{I}\left[D_{\alpha} V, V\right]^{J}+\frac{1}{16} f_{I J a} \Phi^{a} D^{\alpha} V^{I} \bar{D}^{2} D_{\alpha} V^{J}\right\}+\text { h.c. }, \\
& \Delta S_{W}^{3} \supset \int \mathrm{d}^{8} z \frac{1}{3 !} W_{a b c} \Phi^{a} \Phi^{b} \frac{D^{2}}{-4 \square} \Phi^{c}+\text { h.c. }, \quad \Delta S_{F P}^{3} \supset \int \mathrm{d}^{8} z\left(C^{\prime}+\bar{C}^{\prime}\right)_{I}[V, C-\bar{C}]^{I},
\end{aligned}
$$

respectively. The form of the triple interaction involving the Kähler potential has been obtained after extensive use of the consequence of gauge invariance (1). The non-vanishing supergraphs are collected in figure 6.

As we did for the "8" supergraph in subsection 4.2, we will only give the expressions for the resulting scalar integrals of the " $\ominus$ " supergraphs. However, to illustrate how such two loop supergraphs can be evaluated in general, we discuss the supergraph expression of diagram A of figure 6 in some detail. Moreover, this computation shows explicitly how the non-covariance of the result (33) of the " 8 " graph is resolved. The expression for diagram A is given by

$$
i \Gamma_{2 L}^{\mathrm{A}}=\frac{i}{2} \int\left(\mathrm{d}^{8} z\right)_{12} K_{1 a b} \underline{c}^{\underline{c}} \underline{\underline{a}}^{\underline{b}}{ }_{c}\left[\left(\Delta_{\Phi \bar{\Phi}}\right)^{a}{ }_{\underline{a}} \frac{\bar{D}^{2} D^{2}}{16}\right]_{1} \delta_{12}\left[\left(\Delta_{\Phi \bar{\Phi}}\right)_{\underline{b}}^{b} \frac{\bar{D}^{2} D^{2}}{16}\right]_{1} \delta_{12}\left[\left(\Delta_{\Phi \bar{\Phi}}\right)_{\underline{c}}^{c} \frac{D^{2} \bar{D}^{2}}{16}\right]_{1} \delta_{12} .
$$


To reduce this integral to a scalar integral we partially integrate the $\bar{D}^{2} D^{2}$ that acts on the first $\delta_{12}$. Since throughout this work we are only interested in the effective Kähler potential, we systematically ignore all (super covariant) derivatives on background superfield quantities. This implies, that $\bar{D}^{2} D^{2}$ act (in opposite order) on the final $\delta_{12}$ to give: $D^{2} \bar{D}^{2} D^{2} \bar{D}^{2} \delta_{12}=16 \square D^{2} \bar{D}^{2} \delta_{12}$, and hence this supergraph reduces to the scalar integral

$$
i \Gamma_{2 L}^{\mathrm{A}}=\frac{i}{2} \int\left(\mathrm{d}^{4} x\right)_{12} \mathrm{~d}^{4} \theta K_{a b} \underline{\underline{c}} K^{\underline{a} \underline{b}} \underline{c}_{c}\left(\Delta_{\Phi \bar{\Phi}}\right)^{a} \underline{a}_{12} \delta_{12}^{4}\left(\Delta_{\Phi \bar{\Phi}}\right)_{\underline{b}}^{b} \delta_{12}^{4}\left(\Delta_{\Phi \bar{\Phi}}^{\square}\right)^{c} \underline{c}_{12} \delta_{12}^{4} .
$$

Since we are computing the effective potential it is irrelevant whether $K_{a b} \underline{\underline{ }}$ and the mass matrices inside the propagators are functions of coordinate system '1' or '2', hence for notational simplicity we have dropped the coordinate system labels. After the double Wick rotation, transforming to momentum space, and computing the regularized Euclidean integrals, we obtain

$$
\begin{aligned}
& i \Gamma_{2 L}^{\mathrm{A}}=-\frac{i}{2} \int \mathrm{d}^{8} z\left\{K^{\underline{a} \underline{b}}{ }_{c} G^{-1 c}{ }_{\underline{c}} K_{a b} \underline{c}^{\underline{c}} \bar{J}_{\underline{a}}^{a} \underline{b}{ }_{\underline{b}}\left(M^{2}, M^{2}\right)-\bar{\Gamma}^{\underline{a} \underline{b}} \underline{\underline{b}} \bar{W}^{\underline{d} \underline{c}} \Gamma_{a b}{ }_{a b} W_{d c} \bar{I}_{\underline{a}}^{a} \underline{a}_{\underline{b}}{ }^{c} \underline{c}\left(M^{2}, M^{2}, M^{2}\right)\right\} \\
& +i \int \mathrm{d}^{8} z K^{\underline{a}} \underline{\underline{b}}{ }_{c}\left(T_{I} \phi\right)^{c} K_{a b} \underline{\underline{c}}\left(\bar{\phi} T_{J}\right)_{\underline{c}} \bar{I}_{\underline{a}}^{a} \underline{b} \underline{\underline{b}} I J\left(M^{2}, M^{2}, M_{V}^{2}\right) .
\end{aligned}
$$

Here we have used (B.20), and the notation defined in (B.22) and (B.23). In the last term we have used that the Killing vectors $T_{I} \phi$ are perpendicular to the superpotential mass $M_{W}^{2}$, and therefore we may replace the full mass matrix $M^{2}$ by Goldstone boson masses $M_{G}^{2}$. Moreover, by using the relation between the Goldstone boson and the Faddeev-Poppov ghost mass matrices, we can pull out the Killing vectors $\left(T_{I} \phi\right)^{c}$ and $\left(\bar{\phi} T_{J}\right)_{\underline{c}}$, and replace $M_{G}^{2}$ by $M_{C}^{2}$. Finally, because the last term is symmetric in the indices $I, J$, we can replace $M_{C}^{2}$ by the vector multiplet mass matrix $M_{V}^{2}$. The derivation of (36) from (35) illustrates, that even though diagram A has the " $\ominus$ " topology as a supergraph, it turns into a sum of " 8 " and " $\ominus$ " scalar graphs. We see that the first term combines with the contribution (33) of the "8" diagram to form the curvature tensor (4), and therefore leads to a covariant expression as promised. The other parts of diagram A combine with the other " $\ominus$ " diagrams to give rise to covariant expressions as well, as we will demonstrate below.

For the other supergraphs in figure 6 we only give their reduction to scalar graphs and their final compact expressions using the notation $\bar{I}$ and $\bar{J}$, as they can be computed using similar methods as diagram A above. All other supergraphs reduce to scalar graphs of the " $\ominus$ " topology, expect diagram $\mathrm{H}$, which like diagram A, turns into both "8" and " $\ominus$ " scalar graphs. To write down the results in a compact form, we employ the short hand notations

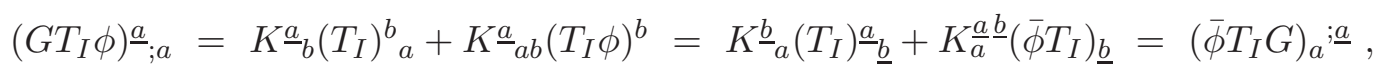

$$
\begin{aligned}
& \left(M_{C}^{2}\right)_{I J} \underline{; a}=2\left(T_{I} G T_{J} \phi\right)^{\underline{a}}+2 K^{\underline{a}} \underline{b} \underline{b}_{b}\left(\bar{\phi} T_{I}\right)_{\underline{b}}\left(T_{J} \phi\right)^{b}, \\
& \left(M_{C}^{2}\right)_{I J ; a}=2\left(\bar{\phi} T_{I} G T_{J}\right)_{a}+2 K^{\underline{b}}{ }_{a b}\left(\bar{\phi} T_{I}\right)_{\underline{b}}\left(T_{J} \phi\right)^{b} .
\end{aligned}
$$

The first equation in (37) is another consequence of gauge invariance (1) of the Kähler potential. Their 
expressions for the other supergraphs of figure 6 as scalar integrals are given by

$$
\begin{aligned}
& i \Gamma_{2 L}^{B}=i \int\left(\mathrm{d}^{4} x\right)_{12} \mathrm{~d}^{4} \theta K_{a b^{\underline{c}}} K^{\underline{a} \underline{b}_{c}}\left(\Delta_{\Phi \bar{\Phi}}\right)^{a}{ }_{\underline{a}} \delta_{12}^{4}\left(\Delta_{\Phi \Phi}\right)^{b c} \delta_{12}^{4}\left(\Delta_{\bar{\Phi} \bar{\Phi}}\right)_{\underline{b}} \delta_{12}^{4},
\end{aligned}
$$

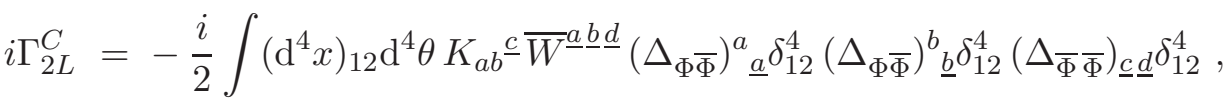

$$
\begin{aligned}
& i \Gamma_{2 L}^{D}=\frac{i}{6} \int\left(\mathrm{d}^{4} x\right)_{12} \mathrm{~d}^{4} \theta W_{a b c} \bar{W}^{\underline{a} \underline{b} \underline{c}}\left(\Delta_{\Phi \bar{\Phi}}\right)^{a}{ }_{\underline{a}} \delta_{12}^{4}\left(\Delta_{\Phi \bar{\Phi}}\right)^{b}{ }_{\underline{b}} \delta_{12}^{4}\left(\Delta_{\Phi \Phi}\right)^{c}{ }_{\underline{\underline{S}}} \delta_{12}^{4}, \\
& i \Gamma_{2 L}^{E}=-i \int\left(\mathrm{d}^{4} x\right)_{12} \mathrm{~d}^{4} \theta\left(G T_{I} \phi\right)^{\underline{a}} ; a\left(\bar{\phi} T_{J} G\right)_{b} ; \underline{b}\left(\Delta_{V}\right)^{I J} \delta_{12}\left(\Delta_{\Phi \bar{\Phi}}\right)^{b}{ }_{a} \delta_{12}^{4}\left(\Delta_{\Phi \bar{\Phi}}\right)^{a}{ }_{b} \delta_{12}^{4}, \\
& i \Gamma_{2 L}^{F}=-i \int\left(\mathrm{d}^{4} x\right)_{12} \mathrm{~d}^{4} \theta K_{a b} \underline{\underline{c}}\left(\bar{\phi} T_{I}\right)_{\underline{c}} K^{\underline{a}} \underline{b}{ }_{c}\left(T_{I} \phi\right)^{c}\left(\Delta_{V}\right)^{I J} \delta_{12}\left(\Delta_{\Phi \bar{\Phi}}\right)^{a}{ }_{\underline{a}} \delta_{12}^{4}\left(\Delta_{\Phi \bar{\Phi}}\right)^{b}{ }_{\underline{b}} \delta_{12}^{4}, \\
& i \Gamma_{2 L}^{G}=-\frac{i}{4} \int\left(\mathrm{d}^{4} x\right)_{12} \mathrm{~d}^{4} \theta c^{I}{ }_{J K} c^{L}{ }_{M N}\left(\Delta_{V}\right)^{M J} \delta_{12}^{4}\left(\left(\Delta_{C}\right)^{K}{ }_{L} \delta_{12}^{4}\left(\Delta_{\bar{C}}\right)^{N}{ }_{I} \delta_{12}^{4}+\right. \\
& \left.+\left(\Delta_{\bar{C}}\right)^{K}{ }_{L} \delta_{12}^{4}\left(\Delta_{C}\right)^{N}{ }_{I} \delta_{12}^{4}-\left(\Delta_{C}\right)^{K}{ }_{L} \delta_{12}^{4}\left(\Delta_{C}\right)^{N}{ }_{I} \delta_{12}^{4}-\left(\Delta_{\bar{C}}\right)^{K}{ }_{L} \delta_{12}^{4}\left(\Delta_{\bar{C}}\right)^{N}{ }_{I} \delta_{12}^{4}\right), \\
& i \Gamma_{2 L}^{H}=\frac{i}{4} \int\left(\mathrm{d}^{4} x\right)_{12} \mathrm{~d}^{4} \theta f_{I J a} f_{K L} \underline{\underline{a}}\left(\Delta_{V} \partial_{m}\right)_{1}^{I K} \delta_{12}^{4}\left(\Delta_{V} \partial^{m}\right)_{1}^{J L} \delta_{12}^{4}\left(\Delta_{\Phi \bar{\Phi}}\right)_{1}^{a} \underline{a} \delta_{12}^{4}, \\
& i \Gamma_{2 L}^{I}=\frac{i}{2} \int\left(\mathrm{d}^{4} x\right)_{12} \mathrm{~d}^{4} \theta f_{I J a}\left(M_{C}^{2}\right)_{K L}{ }^{; \underline{a}}\left(\Delta_{V}\right)_{1}^{I K} \delta_{12}^{4}\left(\Delta_{V}\right)_{1}^{J L} \delta_{12}^{4}\left(\Delta_{\Phi \bar{\Phi}}\right)_{1}^{a} \underline{a} \delta_{12}^{4} . \\
& i \Gamma_{2 L}^{J}=i \int\left(\mathrm{d}^{4} x\right)_{12} \mathrm{~d}^{4} \theta h_{L P} c^{P}{ }_{I N} h_{J Q} c^{Q}{ }_{K M}\left(\Delta_{V}\right)_{1}^{I J} \delta_{12}^{4}\left(\Delta_{V}\right)_{1}^{K L} \delta_{12}^{4}\left(\Delta_{V}\right)_{1}^{M N} \delta_{12}^{4} .
\end{aligned}
$$

Notice that there are also the Hermitian conjugate diagrams of diagrams C and I.

These expressions can be evaluated further using the same Fourier transforms and Wick rotations as employed for diagram A. The diagrams B, C, $\overline{\mathrm{C}}$ and $D$ are all proportional the same integral, hence we can write

$$
\begin{aligned}
i \Gamma_{2 L}^{\mathrm{B}}+i \Gamma_{2 L}^{\mathrm{C}}+i \Gamma_{2 L}^{\overline{\mathrm{C}}}+i \Gamma_{2 L}^{\mathrm{D}}= & \frac{i}{6} \int \mathrm{d}^{8} z\left(\bar{W}^{\underline{a} \underline{b}} \underline{c} W_{a b c}-3 \bar{W}^{\underline{a} \underline{b} \underline{c}} \Gamma_{a b}^{d} W_{d c}-3 \bar{\Gamma}^{\underline{a}} \underline{\underline{b}} \bar{W}^{\underline{d} \underline{c}} W_{a b c}+\right. \\
& \left.+6 \bar{\Gamma}^{\underline{a} \underline{b}} \underline{\underline{d}} \bar{W}^{\underline{d} \underline{c}} \Gamma_{a b}^{d} W_{d c}\right) \bar{I}^{a}{ }_{\underline{a}}^{b} \underline{b} \underline{\underline{b}} \underline{c}\left(M^{2}, M^{2}, M^{2}\right) .
\end{aligned}
$$

Observe that this combines with the parts of (36) that proportional to the double derivatives of the superpotential to form the square of the triple covariant derivative of the superpotential (5). From diagram $\mathrm{E}$ we get the contribution:

$$
i \Gamma_{2 L}^{\mathrm{E}}=-i \int \mathrm{d}^{8} z\left(G T_{I} \phi\right)^{\underline{a}} ; b\left(\bar{\phi} T_{J} G\right)_{a}^{;} \underline{\underline{b}} \bar{I}_{\underline{a}}^{a} \underline{b}_{\underline{b}} I J\left(M^{2}, M^{2}, M_{V}^{2}\right) .
$$

It is not difficult to see that diagram $\mathrm{F}$, given by

$$
i \Gamma_{2 L}^{\mathrm{F}}=-i \int \mathrm{d}^{8} z K^{\underline{a}} \underline{b}_{c}\left(T_{I} \phi\right)^{c} K_{a b} \underline{\underline{c}}\left(\bar{\phi} T_{J}\right)_{\underline{c}} \bar{I}_{\underline{a}}^{a} \underline{b}_{\underline{b}}{ }^{I J}\left(M^{2}, M^{2}, M_{V}^{2}\right)
$$


is precisely opposite to the last contribution of diagram $\mathrm{A}$ in (36), so that these contributions precisely cancel each other and covariance of the combined expression has become manifest. The effect of the ghosts results in diagram $\mathrm{G}$

$$
i \Gamma_{2 L}^{\mathrm{G}}=\frac{i}{2} \int \mathrm{d}^{8} z h_{L P} c^{P}{ }_{I N} h_{J Q} c^{Q} K M\left\{\bar{I}^{I J K L M N}\left(M_{C}^{2}, M_{C}^{2}, M_{V}^{2}\right)-\bar{I}^{I J K L M N}\left(M_{C}^{2}, M_{C}^{2 T}, M_{V}^{2}\right)\right\} .
$$

To arrive at this expression we exploited symmetries of the gauge indices. Like diagram A, diagram $\mathrm{H}$ also contains derivatives $\partial_{m}$ in the numerator, and therefore also this " $\ominus$ " supergraphs becomes a sum of "8" and " $\ominus$ " scalar graphs. The difference is that in this case they act on two different delta functions. We make use of (B.21) to obtain after some algebra

$$
\begin{aligned}
i \Gamma_{2 L}^{\mathrm{H}}= & \frac{i}{8} \int \mathrm{d}^{8} z f_{I K a} \bar{f}_{J L} \underline{a}\left\{2 h^{-1 K L} \bar{J}_{\underline{a}}^{a}{ }_{\underline{a}}^{I J}\left(M^{2}, M_{V}^{2}\right)-G_{\underline{a}}^{-1 a} \bar{J}^{I J K L}\left(M_{V}^{2}, M_{V}^{2}\right)+\right. \\
& \left.+\left(T_{M} \phi\right)^{a}\left(\bar{\phi} T_{N}\right)_{\underline{a}} \bar{I}^{I J K L M N}\left(M_{V}^{2}, M_{V}^{2}, M_{C}^{2}\right)\right\}+\frac{i}{8} \int \mathrm{d}^{8} z\left\{f_{I K b}\left(G^{-1} \bar{W}\right)^{b \underline{a}} \bar{f}_{J L} \underline{b}\left(G^{-1}{ }^{T} W\right)_{\underline{b} a}+\right. \\
& \left.-f_{M K a} \bar{f}_{N L} \underline{a}\left(\delta^{M}{ }_{I}\left(h^{-1} M_{V}^{2}\right)^{N}{ }_{J}+\delta^{N}{ }_{J}\left(h^{-1} M_{V}^{2}\right)^{M}{ }_{I}\right)\right\} \bar{I}_{\underline{a}}^{a}{ }_{\underline{a}}^{I J K L}\left(M^{2}, M_{V}^{2}, M_{V}^{2}\right)
\end{aligned}
$$

Diagram I becomes

$$
i \Gamma_{2 L}^{\mathrm{I}}=\frac{i}{2} \int \mathrm{d}^{8} z f_{I K a}\left(M_{C}^{2}\right)_{J L} \underline{\underline{a}} \bar{I}_{\underline{a}}^{a} I J K L\left(M^{2}, M_{V}^{2}, M_{V}^{2}\right) .
$$

Of course we also have the Hermitian conjugate of this graph. Finally, we have

$$
i \Gamma_{2 L}^{\mathrm{J}}=i \int \mathrm{d}^{8} z h_{L P} c^{P}{ }_{I N} h_{J Q} c^{Q}{ }_{K M} \bar{I}^{I J K L M N}\left(M_{V}^{2}, M_{V}^{2}, M_{V}^{2}\right) .
$$

This complete our calculation of the contributions to the two loop Kähler potential, in the next and final subsection we collect the various contributions together.

\subsection{Summary results for the effective Kähler potential at two loops}

We collect and combine all contributions to the two loop effective Kähler potential obtained in the previous subsections. These contributions result from the two loop graphs depicted in figures 5 and 6. We use those diagrams to refer the different contributions. The full two loop corrections to the Kähler potential can be divided into two parts depending on whether they are only present when the gauge kinetic function is constant or not:

$$
K_{2 L}=K_{2 L}^{\text {universal }}+K_{2 L}^{\text {gauge kinetic }} .
$$

The part of the two loop correction that is present even when the superpotential is constant we call "universal", and the part that is only present for non-constant gauge kinetic functions we call "gauge kinetic". Before we below describe each of these contributions separately, we would like to remind the reader that these results are obtained for perpendicular $M_{W}^{2}$ and $M_{G}^{2}$, because we assumed that the background equations of motion are satisfied. For field configurations that do not, there can be additional corrections. 
To write down the results in a compact way, we use the short hand notations $\bar{J}$ and $\bar{I}$ for the divergent integrals (B.1) and (B.4) containing matrix valued masses with the subdivergences and poles removed. These integrals are evaluated in appendix B, and their expressions are given in (B.22) and (B.23), respectively. As discussed in appendix B.4 it is in principle straightforward to generalize the results for two loop scalar integral with scalar masses to mass matrices. The only subtlety is that the function $\bar{I}^{a} \underline{a}_{\underline{b}}^{b} \underline{\underline{b}} \underline{c}$ takes a sightly different forms depending on the values of the masses due to threshold effects: The functions $\kappa\left(\bar{x}_{i}\right)$ inside $\bar{I}$, see (B.23), depend on whether the scalar quantity $\left(N^{2}\right)^{2}$, defined in (B.9), is positive or negative. As this is a condition on scalar masses, to give an completely explicit expression for $\bar{I}_{\underline{a}}^{a} \underline{b}_{\underline{b}} \underline{c}$, one needs to go to a basis in which the mass matrices are diagonal. It is therefore beneficial to be able to diagonalize the mass matrices explictly. (In the examples discussed in the next section we can easily go to the diagonal basis to perform all computations.) Since we represent all the results here in terms of the integrals $\bar{J}^{a}{ }_{a}^{b} \underline{b}$ and $\bar{I}^{a}{ }_{\underline{a}}^{b}{ }^{b} \underline{b} \underline{c}$, this issue is not explicitly visible in our expressions.

The universal part of the two loop Kähler potential takes the form

$$
\begin{aligned}
& K_{2 L}^{\text {universal }}=\frac{1}{2} R^{\underline{a}}{ }_{a}^{\underline{b}}{ }_{b} \bar{J}_{\underline{a}}^{a} \underline{b}{ }_{b}^{b}\left(M^{2}, M^{2}\right)+\frac{1}{6} \bar{W}^{; \underline{a} \underline{b} \underline{c}} W_{; a b c} \bar{I}_{\underline{a}}^{a} \underline{b}_{\underline{b}} \underline{c} \underline{c}\left(M^{2}, M^{2}, M^{2}\right)+ \\
& +\frac{1}{2} h_{L P} c^{P}{ }_{I N} h_{J Q} c^{Q} K M\left\{\bar{I}^{I J K L M N}\left(M_{C}^{2}, M_{C}^{2}, M_{V}^{2}\right)-\bar{I}^{I J K L M N}\left(M_{C}^{2}, M_{C}^{2 T}, M_{V}^{2}\right)\right\}+ \\
& +h_{L P} c^{P}{ }_{I N} h_{J Q} c^{Q}{ }_{K M} \bar{I}^{I J K L M N}\left(M_{V}^{2}, M_{V}^{2}, M_{V}^{2}\right)-\left(G T_{I} \phi\right)^{\underline{a}} ; b\left(\bar{\phi} T_{J} G\right)_{a} ; \underline{\underline{b}} \bar{I}_{\underline{a}}^{a} \underline{b}_{\underline{b}}^{I J}\left(M^{2}, M^{2}, M_{V}^{2}\right) \text {. }
\end{aligned}
$$

The first term that is proportional to the curvature (4) results from the " 8 " diagram combined with a part of diagram A. The next term depends on the triple covariant derivatives of the superpotential (5). It results from another part of diagram A together with the diagrams B, C, $\bar{C}$ and D. The final part of diagram A cancels diagram $\mathrm{F}$, which involve the exchange of a vector multiplet, and therefore leaves no trace in (47). The second line is a consequence of the ghost diagram G, and the third line is due to the graphs $\mathrm{J}$ and $\mathrm{E}$ in which a vector multiplet is exchanged.

This result is manifestly covariant under diffeomorphisms that preserve the Kähler structure, because it is written in terms of the curvature tensor (4) and the triple covariant derivative of the superpotential (5). The fact that the " 8 " supergraph in figure 5 and the supergraphs A to B of figure 6 combine to covariant expressions provides an important cross check of our two loop computation. Diagram D has been computed in refs. [19, 20, 21, 22] for the renormalizable Wess-Zumino model. The combination of the diagrams " 8 " and A-D have been computed in ref. $[23,24]$ again for a single ungauged chiral multiplet. (However, there seemed to be some differences with our results, in particular that result is not covariant.) In subsection 5.1 we discuss the non-renormalizable Wess-Zumino model, to make the comparison with our results easier. 
When the gauge kinetic function is not constant we find the additional contributions

$$
\begin{aligned}
& K_{2 L}^{\text {gauge kinetic }}=\frac{1}{8} f_{I K a} \bar{f}_{J L} \underline{a}\left\{2 h^{-1 K L} \bar{J}_{\underline{a}}^{a}{ }_{\underline{a}}^{I J}\left(M^{2}, M_{V}^{2}\right)-G_{\underline{a}}^{-1 a} \bar{J}^{I J K L}\left(M_{V}^{2}, M_{V}^{2}\right)+\right. \\
& \left.+\left(T_{M} \phi\right)^{a}\left(\bar{\phi} T_{N}\right)_{\underline{a}} \bar{I}^{I J K L M N}\left(M_{V}^{2}, M_{V}^{2}, M_{C}^{2}\right)\right\}+\frac{1}{8}\left\{f_{I K b}\left(G^{-1} \bar{W}\right)^{b \underline{a}} \bar{f}_{J L} \underline{b}^{-}\left(G^{-1^{T}} W\right)_{\underline{b} a}+\right. \\
& \left.-f_{M K a} \bar{f}_{N L} \underline{a}\left(\delta^{M}{ }_{I}\left(h^{-1} M_{V}^{2}\right)^{N}{ }_{J}+\delta^{N}{ }_{J}\left(h^{-1} M_{V}^{2}\right)^{M}{ }_{I}\right)\right\} \bar{I}_{\underline{a}}^{a} I J K L\left(M^{2}, M_{V}^{2}, M_{V}^{2}\right)+ \\
& +\frac{1}{2}\left(f_{I K a}\left(M_{C}^{2}\right)_{J L} \underline{i}+\bar{f}_{I K} \underline{a}\left(M_{C}^{2}\right)_{J L ; a}\right) \bar{I}_{\underline{a}}^{a}{ }^{I J K L}\left(M^{2}, M_{V}^{2}, M_{V}^{2}\right) .
\end{aligned}
$$

The terms that are proportional to the product of tensors $f$ and $\bar{f}$ arise from diagram $\mathrm{H}$. The last line is the effect of diagram I and it's Hermitian conjugate.

\section{$5 \quad$ Simple applications}

In this section we illustrate our general formulae for the effective Kähler potential at one and two loops, which were given in (28) and (47), (48), respectively, by applying them to some simple supersymmetric models. As discussed in the summary section 4.4, the two loop results are expressed in terms of the tensor integrals $\bar{J}$ and $\bar{I}$; their explicit forms can be found in appendix B. In subsection 5.1 we consider as a first example a general non-renormalizable Wess-Zumino model, and its simplification to the renormalizable version (most previous investigations in the literature only consider this case). Our second example, Super Quantum Electrodynamics, is discussed in subsection 5.2.

\subsection{The (non-)renormalizable Wess-Zumino model}

We consider a single chiral multiplet $\phi$, described by a Kähler potential $K=K(\bar{\phi}, \phi)$ and a superpotential $W(\phi)$. The metric, connection and curvature read

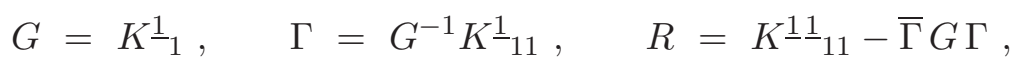

where 1 and $\underline{1}$ denote the differentiation w.r.t. $\phi$ and $\bar{\phi}$, respectively. The triple covariant derivative of the superpotential and the superpotential mass are given by

$$
W_{; 111}=W_{111}-3 \Gamma W_{11}, \quad M_{W}^{2}=G^{-2}\left|W_{11}\right|^{2} .
$$

Hence the one and two loop corrections to the effective Kähler potential read

$$
K_{1 L}=\frac{1}{16 \pi^{2}} \frac{1}{2} M_{W}^{2}\left(2-\ln \frac{M_{W}^{2}}{\bar{\mu}^{2}}\right), \quad K_{2 L}=\frac{1}{2} R G^{-2} \bar{J}+\frac{1}{6}\left|W_{; 111}\right|^{2} G^{-3} \bar{I},
$$

with the short hand notations

$$
\begin{aligned}
\bar{J} & =\frac{1}{\left(16 \pi^{2}\right)^{2}}\left(M_{W}^{2}\right)^{2}\left(1-\ln \frac{M_{W}^{2}}{\bar{\mu}^{2}}\right)^{2}, \\
\bar{I} & =\frac{1}{\left(16 \pi^{2}\right)^{2}} \frac{3}{2} M_{W}^{2}\left[-5+4 \ln \frac{M_{W}^{2}}{\bar{\mu}^{2}}-\ln ^{2} \frac{M_{W}^{2}}{\bar{\mu}^{2}}+12 \kappa(\bar{x})\right] .
\end{aligned}
$$


As we discussed in summary subsection 4.4 the form of $\kappa(\bar{x})$ depends on the sign of $\left(N^{2}\right)^{2}$ defined in B.9. Since $\left(N^{2}\right)^{2}=-\frac{3}{4}\left(M_{W}^{2}\right)^{2}$ is negative, $\kappa(\bar{x})$ is given by (B.19). Using (B.12) we find that $\bar{x}=\frac{4}{3} \sqrt{3}$.

Of particular interest is the reduction to the renormalizable Wess-Zumino model which we use in section 3 to obtain an independent cross check of our one loop result. In this case the Kähler potential is trivial: $K=\bar{\phi} \phi$, which means that the connection and curvature are all zero. The superpotential is given by

$$
W(\phi)=\frac{1}{2} m \phi^{2}+\frac{1}{3 !} \lambda \phi^{3},
$$

with $m$ and $\lambda$ complex parameters, so that the mass $M_{W}^{2}=|m+\lambda \phi|^{2}$. Hence the expressions for the one and two loop Kähler potentials further simplify to

$$
\begin{aligned}
& K_{1 L}=\frac{1}{16 \pi^{2}} \frac{1}{2} M_{W}^{2}\left(2-\ln \frac{M_{W}^{2}}{\bar{\mu}^{2}}\right) \\
& K_{2 L}=\frac{1}{\left(16 \pi^{2}\right)^{2}} \frac{1}{4} M_{W}^{2}\left\{-5+4 \ln \frac{M_{W}^{2}}{\bar{\mu}^{2}}-\ln ^{2} \frac{M_{W}^{2}}{\bar{\mu}^{2}}+12 \kappa(\bar{x})\right\} .
\end{aligned}
$$

\subsection{Super Quantum Electrodynamics}

The theory of Super Quantum Electrodynamics consists of two oppositely charged chiral multiplets $\phi_{+}$and $\phi_{-}$under a U(1) gauge symmetry of which $V$ is the vector superfield. The Kähler potential for this model has the well known form

$$
K=\phi_{+} e^{2 V} \phi_{+}+\phi_{-} e^{-2 V} \phi_{-}, \quad W=0 ;
$$

we assume that super electron is massless to ensure that $M_{W}^{2}$ and $M_{G}^{2}$ are perpendicular automatically. A Fayet-Iliopoulos term can be included, but as this does not affect the results given below, we have not done so here. The gauge kinetic action reads

$$
S_{G}=\frac{1}{4 g^{2}} \int \mathrm{d}^{6} z \mathcal{W}^{\alpha} \mathcal{W}_{\alpha}+\text { h.c. }
$$

where $g^{-2}=h=f$ is the inverse gauge coupling.

Before we get to the explicit formulae for the one and two loop results, we develop some properties of the masses matrices that appear in those expressions. In the following it turns out to be convenient to use the vector and matrix notation

$$
\phi=\left(\begin{array}{c}
\phi_{+} \\
\phi_{-}
\end{array}\right), \quad \bar{\phi}=\left(\begin{array}{ll}
\bar{\phi}_{+} & \bar{\phi}_{-}
\end{array}\right), \quad T=\left(\begin{array}{cc}
1 & 0 \\
0 & -1
\end{array}\right),
$$

for the electron superfields and the charge operator $T$. Because the theory is Abelian the vector and ghost mass parameters are equal

$$
M_{V}^{2}=M_{C}^{2}=2 \bar{\phi} \phi .
$$

The superpotential mass is by definition zero and for Goldstone boson mass matrices we obtain

$$
M_{W}^{2}=0, \quad M_{G}^{2}=2 g^{2}\left(\begin{array}{cc}
\phi_{+} \bar{\phi}_{+} & -\phi_{+} \bar{\phi}_{-} \\
-\phi_{-} \bar{\phi}_{+} & \phi_{-} \bar{\phi}_{-}
\end{array}\right)=g^{2} M_{V}^{2} P_{+} .
$$


The mass matrices can be expressed in term of the Hermitian projection operators $P_{+}$and $P_{-}$which are mutual perpendicular. These projectors

$$
P_{+}=\frac{1}{\bar{\phi} \phi}\left(\begin{array}{c}
\phi_{+} \\
-\phi_{-}
\end{array}\right)\left(\begin{array}{ll}
\bar{\phi}_{+} & -\bar{\phi}_{-}
\end{array}\right), \quad P_{-}=\frac{1}{\bar{\phi} \phi}\left(\begin{array}{c}
\bar{\phi}_{-} \\
\bar{\phi}_{+}
\end{array}\right)\left(\begin{array}{ll}
\phi_{-} & \phi_{+}
\end{array}\right)
$$

diagonalize the total mass matrix $M^{2}$ since

$$
M^{2}=M_{W}^{2}+M_{G}^{2}=m_{+}^{2} P_{+}+m_{-}^{2} P_{-},
$$

with the mass eigenvalues $m_{+}^{2}=g^{2} M_{V}^{2}$ and $m_{-}^{2}=0$. Because of the properties of these projection operators we can express the tensor $\bar{I}_{\underline{a}}^{a} \underline{b} \underline{b}\left(M^{2}, M^{2}, g^{2} M_{V}^{2}\right)$, for the U(1) theory, as a sum of scalar integrals $\bar{I}$ :

$$
\bar{I}_{\underline{a} \underline{b}}^{a b}\left(M^{2}, M^{2}, g^{2} M_{V}^{2}\right)=\sum_{r, s}\left(P_{r}\right)^{a}{ }_{\underline{a}}\left(P_{s}\right)^{b} \underline{\underline{b}} \bar{I}\left(m_{r}^{2}, m_{s}^{2}, g^{2} M_{V}^{2}\right)
$$

where the sum is over $r, s=+,-$. This shows that the evaluation of the two loop result reduces computing the traces $\operatorname{tr}\left(P_{r} T P_{s} T\right)$ because $(G T \phi)^{\underline{a}} ; a=T^{a}{ }_{a}$, and $(\bar{\phi} T G)_{b} ; \underline{b}=T^{\underline{b}}$. Explicitly we find for these traces

$$
\operatorname{tr}\left(P_{+} T P_{+} T\right)=\operatorname{tr}\left(P_{-} T P_{-} T\right)=\left(\frac{\bar{\phi} \sigma_{3} \phi}{\bar{\phi} \phi}\right)^{2}, \quad \operatorname{tr}\left(P_{+} T P_{-} T\right)=\left|\frac{\phi^{T} \sigma_{1} \phi}{\bar{\phi} \phi}\right|^{2},
$$

with $\sigma_{1}$ and $\sigma_{3}$ the standard Pauli matrices.

After this exposition it is not difficult to see that the one and two loop corrections to the effective Kähler potential are given by the following expressions. At the one loop level we find

$$
K_{1 L}=-\frac{1}{16 \pi^{2}} g^{2} M_{V}^{2}\left(2-\ln \frac{g^{2} M_{V}^{2}}{\bar{\mu}^{2}}\right) .
$$

We have dropped the contribution coming from the superpotential mass matrix as it gives a mere constant which is irrelevant for the Kähler geometry. The two loop result takes the form

$$
K_{2 L}=-\left\{\bar{I}\left(m_{+}^{2}, m_{+}^{2}, g^{2} M_{V}^{2}\right)+\bar{I}\left(m_{-}^{2}, m_{-}^{2}, g^{2} M_{V}^{2}\right)\right\}\left(\frac{\bar{\phi} \sigma_{3} \phi}{\bar{\phi} \phi}\right)^{2}-2 \bar{I}\left(m_{+}^{2}, m_{-}^{2}, g^{2} M_{V}^{2}\right)\left|\frac{\phi^{T} \sigma_{1} \phi}{\bar{\phi} \phi}\right|^{2} .
$$

\section{A One loop scalar integrals}

This appendix is devoted to the evaluation of some one loop scalar integrals that arise in the main text of this paper. We compute these scalar integrals in the $\overline{\mathrm{MS}}$ scheme: We evaluate the integrals in $D=4-2 \epsilon$ dimensions, and we introduce the renormalization scale $\mu$ such that all $D$ dimensional integrals have the same mass dimensions as their divergent four dimensional counter parts. Even though these one loop integrals are well-known, we feel that they should be collected here, because their importance for the subtraction of the subdivergences at the two loop level. Moreover, this allows us to introduce some notation for renormalized quantities that we are employing throughout this work.

At the one loop level we encounter three different types of integrals. The first integral reads

$$
J\left(m^{2}\right)=\int \frac{\mathrm{d}^{D} p}{(2 \pi)^{D} \mu^{D-4}} \frac{1}{p^{2}+m^{2}}=-\frac{m^{2}}{16 \pi^{2}}\left(4 \pi \frac{\mu^{2}}{m^{2}}\right)^{2-\frac{D}{2}} \frac{\Gamma\left(2-\frac{D}{2}\right)}{\frac{D}{2}-1} .
$$


For the applications to two loop graphs we need the expansion of this integral to first order in $\epsilon$ :

$$
J\left(m^{2}\right)=-\frac{m^{2}}{16 \pi^{2}}\left[\frac{1}{\epsilon}+1-\ln \frac{m^{2}}{\bar{\mu}^{2}}+\epsilon\left(1+\frac{1}{2} \zeta(2)-\ln \frac{m^{2}}{\bar{\mu}^{2}}+\frac{1}{2} \ln ^{2} \frac{m^{2}}{\bar{\mu}^{2}}\right)\right] .
$$

Here we have introduced the $\overline{\mathrm{MS}}$ scale $\bar{\mu}^{2}=4 \pi e^{-\gamma} \mu^{2}$ with the Euler constant $\gamma$ and $\zeta(2)=\frac{\pi^{2}}{6}$. The other two integrals

$$
L\left(m^{2}\right)=\int \frac{\mathrm{d}^{D} p}{(2 \pi)^{D} \mu^{D-4}} \frac{1}{p^{2}} \ln \left(1+\frac{m^{2}}{p^{2}}\right)=\frac{m^{2}}{16 \pi^{2}}\left(4 \pi \frac{\mu^{2}}{m^{2}}\right)^{2-\frac{D}{2}} \frac{\Gamma\left(2-\frac{D}{2}\right)}{\left(\frac{D}{2}-1\right)^{2}}
$$

and

$$
S\left(m^{2}\right)=\int \frac{\mathrm{d}^{D} p}{(2 \pi)^{D} \mu^{D-4}} \frac{1}{\left(p^{2}+m^{2}\right)^{2}}=\frac{1}{16 \pi^{2}}\left(4 \pi \frac{\mu^{2}}{m^{2}}\right)^{2-\frac{D}{2}} \Gamma\left(2-\frac{D}{2}\right)
$$

are only relevant for one loop computations in this work, therefore we only need to expand them to zeroth order in $\epsilon$ :

$$
L\left(m^{2}\right)=\frac{m^{2}}{16 \pi^{2}}\left[\frac{1}{\epsilon}+2-\ln \frac{m^{2}}{\bar{\mu}^{2}}\right], \quad S\left(m^{2}\right)=\frac{1}{16 \pi^{2}}\left[\frac{1}{\epsilon}-\ln \frac{m^{2}}{\bar{\mu}^{2}}\right] .
$$

\section{B Two loop scalar integrals}

At the two loop level we encounter two different scalar momentum integrals. As can be seen in figure 4 they have the topology of an " 8 " (figure 4.a) and " $\ominus$ " (figure 4.b). (The third topology depicted in figure 4.c is the same as figure 4.a as a momentum integral, and can therefore be disregarded in this appendix.) Being two loop graphs, these integrals contain subdivergences. The subtraction of subdivergences is crucial, because otherwise one would not obtain local counter terms from the two loop level onwards. A generic feature of such non-local counter terms is, that they involve terms like $\frac{1}{\epsilon} \ln \bar{\mu}^{2}$. In our calculation such terms are also absent after all subdivergences are subtracted off. In this work we take the practical approach that these subdivergences can be subtracted off on a diagram by diagram level directly, instead of computing explicitly one loop graphs with one loop counter terms inserted. The expression of a two loop integral $I$ with all its subdivergences subtracted off is denoted by $\hat{I}$.

\section{B.1 The scalar "8" integral}

The "8" graph is easy to evaluate as it is the product of two one loop integrals defined in (A.1):

$$
J\left(m_{1}^{2}, m_{2}^{2}\right)=J\left(m_{1}^{2}\right) J\left(m_{2}^{2}\right) .
$$

The subtraction of the subdivergences leads to

$$
\hat{J}\left(m_{1}^{2}, m_{2}^{2}\right)=J\left(m_{1}^{2}, m_{2}^{2}\right)+\frac{1}{16 \pi^{2}} \frac{1}{\epsilon}\left(m_{2}^{2} J\left(m_{1}^{2}\right)+m_{1}^{2} J\left(m_{2}^{2}\right)\right) .
$$

Expanding this to zeroth order in $\epsilon$ gives

$$
\hat{J}\left(m_{1}^{2}, m_{2}^{2}\right)=\frac{m_{1}^{2} m_{2}^{2}}{\left(16 \pi^{2}\right)^{2}}\left[-\frac{1}{\epsilon^{2}}+\left(1-\ln \frac{m_{1}^{2}}{\bar{\mu}^{2}}\right)\left(1-\ln \frac{m_{2}^{2}}{\bar{\mu}^{2}}\right)\right] .
$$

We refer to this expression as $\bar{J}\left(m_{1}^{2}, m_{2}^{2}\right)$, when the pole in $\epsilon^{2}$ is subtracted off. 


\section{B.2 The basic scalar " $\ominus$ " diagram}

The evaluation of the " $\ominus$ " graph is much more involved when all three propagator lines correspond to three different masses $m_{1}^{2}, m_{2}^{2}$ and $m_{3}^{2}$ :

$$
I\left(m_{1}^{2}, m_{2}^{2}, m_{3}^{2}\right)=\int \frac{\mathrm{d}^{D} p \mathrm{~d}^{D} q}{(2 \pi)^{2 D} \mu^{2(D-4)}} \frac{1}{p^{2}+m_{1}^{2}} \frac{1}{q^{2}+m_{2}^{2}} \frac{1}{(p+q)^{2}+m_{3}^{2}} .
$$

To compute this integral directly is difficult; various methods to do so can be found in the literature $[36,37,38,39,40,41]$. These results generically lead to complicated expressions, or are only valid in specific limits or certain simplifying assumptions. In particular, the fact that this integral is manifestly symmetric in the masses is generically lost. An elegant indirect way of computing this integral leading to a surprisingly simple result has been presented in refs. [42, 43]. (See also [44].) For completeness we review their method of characteristics.

The essential idea of the method of characteristics is, instead of computing the integral (B.4) head on, to relate the expression of this integral for various values of the masses to each other. In this way the complicated initial integral can be expressed in terms of a combination of simpler integrals. In particular, when two of the three masses are zero, the evaluation of the integral can be performed by standard methods directly:

$$
I\left(m^{2}, 0,0\right)=\frac{m^{2}}{\left(16 \pi^{2}\right)^{2}}\left(4 \pi \frac{\mu^{2}}{m^{2}}\right)^{4-D} \frac{\Gamma(3-D) \Gamma\left(\frac{D}{2}-1\right)^{2} \Gamma\left(2-\frac{D}{2}\right)}{\Gamma\left(\frac{D}{2}\right)} .
$$

Therefore we seek to relate $I\left(m_{1}^{2}, m_{2}^{2}, m_{3}^{2}\right)$ to $I\left(M_{1}^{2}, M_{2}^{2}, 0\right)$ and then that one to $I\left(2 N^{2}, 0,0\right)$. (The masses $M_{1}^{2}, M_{2}^{2}$ and $N^{2}$ will be determined below.)

To related the expressions of integral (B.4) for different values of $m_{1}^{2}, m_{2}^{2}$ and $m_{3}^{2}$ to each other means that we would like to describe a flow through the space of these masses. Such a flow can be encoded by a partial differential that the integral (B.4) satisfies. Of course this equation should be sufficiently simple to be of practical use. For the case at hand a convenient partial differential equation is given by

$$
\left[\left(m_{1}^{2}-m_{2}^{2}\right) \frac{\partial}{\partial m_{3}^{2}}+\text { cycl. }\right] I\left(m_{1}^{2}, m_{2}^{2}, m_{3}^{2}\right)=\frac{\partial J\left(m_{3}^{2}\right)}{\partial m_{3}^{2}}\left(J\left(m_{1}^{2}\right)-J\left(m_{2}^{2}\right)\right)+\text { cycl. },
$$

where "+cycl." indicates the summation over the cyclic permutation of the labels 1,2,3 on the masses. This partial differential equation is obtained by combining various equations that can be found by integrating the total derivative

$$
\frac{\partial}{\partial p^{\mu}}\left[p^{\mu} \frac{1}{p^{2}+m_{1}^{2}} \frac{1}{q^{2}+m_{2}^{2}} \frac{1}{(p+q)^{2}+m_{3}^{2}}\right]
$$

over $p$ and $q$, and similar for $\frac{\partial}{\partial q^{\mu}}\left[q^{\mu} \ldots\right]$. Rewriting the result as partial differentiation w.r.t. the masses, and using cyclic permutations of the labels on the masses. Along the lines of flow, described by the equations

$$
\frac{\mathrm{d} m_{1}^{2}}{\mathrm{~d} t}=m_{2}^{2}-m_{3}^{2}, \quad \frac{\mathrm{d} m_{2}^{2}}{\mathrm{~d} t}=m_{3}^{2}-m_{1}^{2}, \quad \frac{\mathrm{d} m_{3}^{2}}{\mathrm{~d} t}=m_{1}^{2}-m_{2}^{2},
$$


this partial differential equation can be integrated. Such a flow line is an arc of a circle defined by the intersection of a plane and a sphere

$$
m_{1}^{2}+m_{2}^{2}+m_{3}^{2}=\bar{m}^{2}, \quad\left(m_{1}^{2}\right)^{2}+\left(m_{2}^{2}\right)^{2}+\left(m_{3}^{2}\right)^{2}=2\left(N^{2}\right)^{2}+\frac{1}{2}\left(\bar{m}^{2}\right)^{2} .
$$

It follows from the flow equations (B.8) that $\bar{m}^{2}$ and $N^{2}$ are constants. If $\left(N^{2}\right)^{2} \geq 0$, it follows that the point $\left(M_{1}^{2}, M_{2}^{2}, M_{3}^{2}=0\right)$, with

$$
M_{1}^{2}=\frac{\bar{m}^{2}}{2}+N^{2}, \quad M_{2}^{2}=\frac{\bar{m}^{2}}{2}-N^{2},
$$

lies on the same circle as the starting point $\left(m_{1}^{2}, m_{2}^{2}, m_{3}^{2}\right)$, and therefore we can obtain a relation between $I\left(m_{1}^{2}, m_{2}^{2}, m_{3}^{2}\right)$ and $I\left(M_{1}^{2}, M_{2}^{2}, 0\right)$ by integrating the partial differential equation (B.6) along this arc. Similarly, we can consider the partial differential equation

$$
\left(M_{2}^{2}-M_{1}^{2}\right)\left(\frac{\partial}{\partial M_{1}^{2}}+\frac{\partial}{\partial M_{2}^{2}}\right) I\left(M_{1}^{2}, M_{2}^{2}, 0\right)=\frac{\partial J\left(M_{2}^{2}\right)}{\partial M_{2}^{2}} J\left(M_{1}^{2}\right)-\frac{\partial J\left(M_{1}^{2}\right)}{\partial M_{1}^{2}} J\left(M_{2}^{2}\right)
$$

for $I\left(M_{1}^{2}, M_{2}^{2}, 0\right)$. In this case the flow is along straight lines, because the right hand side of this equation is also proportional to $\left(M_{2}^{2}-M_{1}^{2}\right)$. Therefore we can relate the integrals $I\left(M_{1}^{2}, M_{2}^{2}, 0\right)$ and $I\left(2 N^{2}, 0,0\right)$, with $2 N^{2}=M_{1}^{2}-M_{2}^{2}$. By combining these results, the expression (B.5), and using the short-hand notations

$$
\bar{m}_{i}^{2}=\frac{\bar{m}^{2}}{2}-m_{i}^{2}, \quad \bar{x}_{i}=\frac{\bar{m}_{i}^{2}}{|N|^{2}}
$$

one can obtain the following factorized form

$$
I\left(m_{1}^{2}, m_{2}^{2}, m_{3}^{2}\right)=-I_{C}\left[F\left(\bar{x}_{2}\right)+F\left(\bar{x}_{3}\right)-F\left(-\bar{x}_{1}\right)-\frac{1}{2} \frac{\Gamma\left(\frac{3}{2}-\frac{D}{2}\right) \Gamma\left(\frac{D}{2}-1\right)}{\Gamma\left(\frac{1}{2}\right)}\right],
$$

where we have identified a common factor

$$
I_{C}=\frac{N^{2}}{\left(16 \pi^{2}\right)^{2}}\left(4 \pi \frac{\mu^{2}}{N^{2}}\right)^{4-D} \frac{\Gamma\left(2-\frac{D}{2}\right)^{2}}{\frac{D}{2}-1}, \quad \text { and } \quad F(\bar{x})=\int_{1}^{\bar{x}} \mathrm{~d} s\left(s^{2}-1\right)^{\frac{D}{2}-2}
$$

is a shorthand for the remaining integral. The expression (B.13) is not manifestly symmetric in all permutations of $\bar{x}_{1}, \bar{x}_{2}, \bar{x}_{3}$. But this symmetry is present because $F(x)-F(-y)=F(y)-F(-x)$ for any $x, y$. In the " $\ominus$ " diagram one can identify three one loop subgraphs, it follows that the subdivergences are removed by defining

$$
\hat{I}\left(m_{1}^{2}, m_{2}^{2}, m_{3}^{2}\right)=I\left(m_{1}^{2}, m_{2}^{2}, m_{3}^{2}\right)-\frac{1}{16 \pi^{2}} \frac{1}{\epsilon}\left(J\left(m_{1}^{2}\right)+J\left(m_{2}^{2}\right)+J\left(m_{3}^{2}\right)\right),
$$

with $J\left(m^{2}\right)$ given in (A.2).

To expand this expression to the zeroth order in $\epsilon$ explicitly, we need to compute various integrals. Most of them are straightforward, except

$$
\begin{aligned}
& \int_{1}^{\bar{x}} \mathrm{~d} s \ln |s+1| \ln |s-1|=\frac{1}{2} \ln ^{2} 2+2 \ln 2-2+2 \bar{x}-\bar{x} \ln \left|\bar{x}^{2}-1\right|+\ln \left|\frac{\bar{x}-1}{\bar{x}+1}\right|+ \\
&+\bar{x} \ln |\bar{x}+1| \ln |\bar{x}-1|+\frac{1}{2} \ln \left|\bar{x}^{2}-1\right| \ln \left|\frac{\bar{x}-1}{\bar{x}+1}\right|-4 \kappa(\bar{x}),
\end{aligned}
$$


with

$$
\kappa(\bar{x})=-\int_{0}^{a} \mathrm{~d} t \ln |\sinh t|-\frac{3}{16} \ln ^{2} 2-\frac{1}{4} \zeta(2), \quad a=\operatorname{coth}^{-1} \bar{x} .
$$

After some algebra we can express the " $\ominus$ " graph with the subdivergences subtracted as

$$
\begin{aligned}
\hat{I}\left(m_{1}^{2}, m_{2}^{2}, m_{3}^{2}\right)= & \frac{1}{2} \frac{1}{\left(16 \pi^{2}\right)^{2}}\left[\bar{m}^{2}\left(\frac{1}{\epsilon^{2}}-\frac{1}{\epsilon}\right)-5 \bar{m}^{2}+4 \sum_{i} m_{i}^{2} \ln \frac{m_{i}^{2}}{\bar{\mu}^{2}}+\right. \\
& \left.-2\left(\bar{m}_{1}^{2} \ln \frac{m_{2}^{2}}{\bar{\mu}^{2}} \ln \frac{m_{3}^{2}}{\bar{\mu}^{2}}+\text { cycl. }\right)+8 N^{2}\left(-\kappa\left(-\bar{x}_{1}\right)+\kappa\left(\bar{x}_{2}\right)+\kappa\left(\bar{x}_{3}\right)\right)\right],
\end{aligned}
$$

where $\bar{x}_{i}$ is given in (B.12). The calculation that we have reviewed here is valid for $\left(N^{2}\right)^{2} \geq 0$, in the opposite case one can show [43] that (B.18) still holds, but now with $N^{2} \rightarrow|N|^{2}$ everywhere even when it appears implicit, $-\kappa\left(-\bar{x}_{1}\right) \rightarrow \kappa\left(\bar{x}_{1}\right)$ and

$$
\kappa(\bar{x})=-\int_{0}^{a} \mathrm{~d} t \ln |\cos t|, \quad a=\tan ^{-1} \bar{x} .
$$

\section{B.3 Other scalar " $\ominus "$ diagrams}

In the computation of the supergraphs in section 4.3 we encounter integrals that are the same as (B.4)

except that the numerator is more complicated. Fortunately, these integrals can be reduced to (B.4) and (B.1). In particular, we have that:

$$
\int \frac{\mathrm{d}^{D} p \mathrm{~d}^{D} q}{(2 \pi)^{2 D} \mu^{2(D-4)}} \frac{p^{2}}{p^{2}+m_{1}^{2}} \frac{1}{q^{2}+m_{2}^{2}} \frac{1}{(p+q)^{2}+m_{3}^{2}}=J\left(m_{2}^{2}, m_{3}^{2}\right)-m_{1}^{2} I\left(m_{1}^{2}, m_{2}^{2}, m_{3}^{2}\right),
$$

and

$$
\begin{gathered}
\int \frac{\mathrm{d}^{D} p \mathrm{~d}^{D} q}{(2 \pi)^{2 D} \mu^{2(D-4)}} \frac{2 p \cdot q}{\left(p^{2}+m_{1}^{2}\right)\left(q^{2}+m_{2}^{2}\right)} \frac{1}{(p+q)^{2}+m_{3}^{2}}=-J\left(m_{2}^{2}, m_{3}^{2}\right)-J\left(m_{1}^{2}, m_{3}^{2}\right)+J\left(m_{1}^{2}, m_{2}^{2}\right) \\
+\left(m_{1}^{2}+m_{2}^{2}-m_{3}^{2}\right) I\left(m_{1}^{2}, m_{2}^{2}, m_{3}^{2}\right) .
\end{gathered}
$$

\section{B.4 Generalization to mass matrices}

In the section 4 we are dealing with the two loop calculation of supersymmetric theories with multiple scalar and vector multiplets, and therefore the masses are matrices rather than simple numbers. Here we define our notation to describe two loop integrals that involve mass matrices with all subdivergences and the $1 / \epsilon$ poles subtracted. For notational convenience we choose to include the inverse metric $G^{-1}$ in these definitions, so that the expressions contain an equal number of holomorphic and antiholomorphic indices.

For the integral $J$, given in (B.1), we obtain a factorized form

$$
\bar{J}_{\underline{a} \underline{b}}^{a b}\left(m_{1}^{2}, m_{2}^{2}\right)=\frac{1}{\left(16 \pi^{2}\right)^{2}}\left[m_{1}^{2}\left(1-\ln \frac{m_{1}^{2}}{\bar{\mu}^{2}}\right) G^{-1}\right]_{\underline{a}}^{a}\left[m_{2}^{2}\left(1-\ln \frac{m_{2}^{2}}{\bar{\mu}^{2}}\right) G^{-1}\right]_{\underline{b}}^{b} .
$$


The expression for the integral $I$, defined in (B.4), with the subdivergences and poles subtracted does not factorize, see (B.18), consequently the matrix generalization is more complicated:

$$
\begin{aligned}
& \bar{I}_{\underline{a} \underline{b} \underline{b} \underline{c}}^{a b}\left(m_{1}^{2}, m_{2}^{2}, m_{3}^{2}\right)=\frac{1}{2} \frac{1}{\left(16 \pi^{2}\right)^{2}}\left\{\left(-\frac{5}{2} m_{1}^{2}+4 m_{1}^{2} \ln \frac{m_{1}^{2}}{\bar{\mu}^{2}} G^{-1}\right)_{\underline{a}}^{a} G^{-1 b}{ }_{\underline{b}} G^{-1 c}{ }_{\underline{c}}+\right. \\
& -G^{-1^{a}}{ }_{\underline{a}}\left(m_{2}^{2} \ln \frac{m_{2}^{2}}{\bar{\mu}^{2}} G^{-1}\right)_{\underline{b}}^{b}\left(\ln \frac{m_{3}^{2}}{\bar{\mu}^{2}} G^{-1}\right)_{\underline{c}}^{c}-G^{-1^{a}}{ }_{\underline{a}}\left(\ln \frac{m_{2}^{2}}{\bar{\mu}^{2}} G^{-1}\right)_{\underline{b}}^{b}\left(m_{3}^{2} \ln \frac{m_{3}^{2}}{\bar{\mu}^{2}} G^{-1}\right)_{\underline{c}}^{c}+ \\
& \left.+\left(m_{1}^{2} G^{-1}\right)_{\underline{a}}^{a}\left(\ln \frac{m_{2}^{2}}{\bar{\mu}^{2}} G^{-1}\right)_{\underline{b}}^{b}\left(\ln \frac{m_{3}^{2}}{\bar{\mu}^{2}} G^{-1}\right)_{\underline{c}}^{c}+\text { cycl. }+ \text { etc. }\right\} \text {. }
\end{aligned}
$$

Here the "+cycl." denote the cyclic permutation of the labels 1,2,3 and the corresponding indices $a, b, c$ and $\underline{a}, \underline{b}, \underline{c}$, and with "+etc." we refer to the expansion of the $N^{2} \sum_{i} \kappa\left(\bar{x}_{i}\right)$ term in (B.18). The functions $\kappa\left(\bar{x}_{i}\right)$ take two different forms ((B.17) or (B.19)) depending on in which regime $\left(\left(N^{2}\right)>0\right.$ or $\left.\left(N^{2}\right)^{2}<0\right)$ they are evaluated. Since this condition is written down for scalar masses, it means that in the case matrices it has to be evaluated in the diagonal basis.

This notation can be extended to included also adjoint indices $I, J, \ldots$ For those we take the convention that all indices are written as superscripts, i.e. these expressions include appropriate powers of the inverse metric $h^{-1}$ defined below (10). The integrals given in appendix B.3 can be reinterpreted in an analogous way as matrix expressions.

\section{References}

[1] R. Jackiw "Functional evaluation of the effective potential" Phys. Rev. D9 (1974) 1686.

[2] G. von Gersdorff and A. Hebecker "Radius stabilization by two-loop casimir energy" Nucl. Phys. B720 (2005) 211-227 [hep-th/0504002].

[3] M. T. Grisaru, W. Siegel, and M. Rocek "Improved methods for supergraphs" Nucl. Phys. B159 (1979) 429.

[4] V. A. Novikov, M. A. Shifman, A. I. Vainshtein, and V. I. Zakharov "Instantons in supersymmetric theories" Nucl. Phys. B223 (1983) 445.

[5] M. A. Shifman and A. I. Vainshtein "Solution of the anomaly puzzle in SUSY gauge theories and the Wilson operator expansion" Nucl. Phys. B277 (1986) 456.

[6] N. Seiberg "Naturalness versus supersymmetric nonrenormalization theorems" Phys. Lett. B318 (1993) 469-475 [hep-ph/9309335].

[7] N. Seiberg "Exact results on the space of vacua of four-dimensional SUSY gauge theories" Phys. Rev. D49 (1994) 6857-6863 [hep-th/9402044].

[8] K. A. Intriligator, R. G. Leigh, and N. Seiberg "Exact superpotentials in four-dimensions" Phys. Rev. D50 (1994) 1092-1104 [hep-th/9403198].

[9] N. Seiberg and E. Witten "Electric - magnetic duality, monopole condensation, and confinement in N=2 supersymmetric Yang-Mills theory" Nucl. Phys. B426 (1994) 19-52 [hep-th/9407087].

[10] M. T. Grisaru, M. Rocek, and R. von Unge "Effective Kähler potentials" Phys. Lett. B383 (1996) 415-421 [hep-th/9605149].

[11] B. de Wit, M. T. Grisaru, and M. Rocek "Nonholomorphic corrections to the one-loop N=2 super YangMills action" Phys. Lett. B374 (1996) 297-303 [hep-th/9601115]. 
[12] A. Pickering and P. C. West "The one loop effective super-potential and non- holomorphicity" Phys. Lett. B383 (1996) 54-62 [hep-th/9604147].

[13] I. L. Buchbinder, M. Cvetic, and A. Y. Petrov "One-loop effective potential of $\mathrm{N}=1$ supersymmetric theory and decoupling effects" Nucl. Phys. B571 (2000) 358-418 [hep-th/9906141].

[14] A. Brignole "One-loop kaehler potential in non-renormalizable theories" Nucl. Phys. B579 (2000) 101-116 [hep-th/0001121].

[15] M. K. Gaillard, V. Jain, and K. Saririan "Supergravity coupled to chiral and Yang-Mills matter at one loop" Phys. Lett. B387 (1996) 520-528 [hep-th/9606135].

[16] M. K. Gaillard "Pauli-Villars regularization of globally supersymmetric theories" Phys. Lett. B347 (1995) 284-290 [hep-th/9412125].

[17] M. K. Gaillard "Pauli-Villars regularization of supergravity coupled to chiral and Yang-Mills matter" Phys. Lett. B342 (1995) 125-131 [hep-th/9408149].

[18] A. Falkowski "On the one-loop kaehler potential in five-dimensional brane-world supergravity" JHEP 05 (2005) 073 [hep-th/0502072].

[19] I. L. Buchbinder, S. M. Kuzenko, and A. Y. Petrov "Superfield chiral effective potential" Phys. Lett. B321 (1994) 372-377.

[20] I. L. Buchbinder, S. Kuzenko, and Z. Yarevskaya "Supersymmetric effective potential: Superfield approach" Nucl. Phys. B411 (1994) 665-692.

[21] I. L. Buchbinder, S. M. Kuzenko, A. Y. Petrov, and Z. V. Yarevskaya "Superfield effective potential" [hep-th/9501047].

[22] I. L. Buchbinder, S. M. Kuzenko, and A. Y. Petrov "Superfield effective potential in the two loop approximation" Phys. Atom. Nucl. 59 (1996) 148-153.

[23] I. L. Buchbinder and A. Y. Petrov "Superfield effective action within the general chiral superfield model" Phys. Atom. Nucl. 63 (2000) 1657-1670.

[24] A. Y. Petrov "Effective action in general chiral superfield model" [hep-th/0002013].

[25] I. L. Buchbinder and S. M. Kuzenko "Ideas and methods of supersymmetry and supergravity: Or a walk through superspace". Bristol, UK: IOP (1998) 656 p.

[26] J. Wess and J. Bagger "Supersymmetry and supergravity". Princeton, USA: Univ. Pr. (1992) 259 p.

[27] S. J. Gates, M. T. Grisaru, M. Rocek, and W. Siegel "Superspace, or one thousand and one lessons in supersymmetry" Front. Phys. 58 (1983) 1-548 [hep-th/0108200].

[28] P. C. West "Introduction to supersymmetry and supergravity". Singapore, Singapore: World Scientific (1990) $425 \mathrm{p}$.

[29] W. Siegel "Supersymmetric dimensional regularization via dimensional reduction" Phys. Lett. B84 (1979) 193.

[30] D. M. Capper, D. R. T. Jones, and P. van Nieuwenhuizen "Regularization by dimensional reduction of supersymmetric and nonsupersymmetric gauge theories" Nucl. Phys. B167 (1980) 479.

[31] I. Jack, D. R. T. Jones, and K. L. Roberts "Equivalence of dimensional reduction and dimensional regularization" Z. Phys. C63 (1994) 151-160 [hep-ph/9401349].

[32] I. Jack and D. R. T. Jones "Regularisation of supersymmetric theories" [hep-ph/9707278].

[33] J. Bagger, E. Poppitz, and L. Randall "Destabilizing divergences in supergravity theories at two loops" Nucl. Phys. B455 (1995) 59-82 [hep-ph/9505244]. 
[34] W. Fischler, H. P. Nilles, J. Polchinski, S. Raby, and L. Susskind "Vanishing renormalization of the D term in supersymmetric U(1) theories" Phys. Rev. Lett. 47 (1981) 757.

[35] S. Weinberg "The quantum theory of fields. Vol. 3: Supersymmetry". Cambridge, UK: Univ. Pr. (2000) $419 \mathrm{p}$.

[36] G. Fogleman, G. D. Starkman, and K. S. Viswanathan "Two loop calculation of the effective potential for the Wess-Zumino model" Phys. Lett. B133 (1983) 393.

[37] G. Fogleman and K. S. Viswanathan "The effective potential for chiral supersymmetric models" Phys. Rev. D30 (1984) 1364.

[38] R. D. C. Miller "The two loop effective potential of the Wess-Zumino model" Nucl. Phys. B241 (1984) 535 .

[39] A. I. Davydychev and J. B. Tausk "Two loop selfenergy diagrams with different masses and the momentum expansion" Nucl. Phys. B397 (1993) 123-142.

[40] A. I. Davydychev, V. A. Smirnov, and J. B. Tausk "Large momentum expansion of two loop selfenergy diagrams with arbitrary masses" Nucl. Phys. B410 (1993) 325-342 [hep-ph/9307371].

[41] M. Misiak and M. Munz "Two loop mixing of dimension five flavor changing operators" Phys. Lett. B344 (1995) 308-318 [hep-ph/9409454].

[42] C. Ford and D. R. T. Jones "The effective potential and the differential equations method for Feynman integrals" Phys. Lett. B274 (1992) 409-414.

[43] C. Ford, I. Jack, and D. R. T. Jones "The Standard Model effective potential at two loops" Nucl. Phys. B387 (1992) 373-390 [hep-ph/0111190].

[44] S. P. Martin "Two-loop effective potential for a general renormalizable theory and softly broken supersymmetry" Phys. Rev. D65 (2002) 116003 [hep-ph/0111209]. 